\title{
GENERALIZED REDUCED-FORM AUCTIONS: A NETWORK-FLOW APPROACH
}

\author{
YEON-KOO CHE, JINWOO KIM, AND KONRAD MIERENDORFF
}

\begin{abstract}
We develop a network-flow approach for characterizing interim-allocation rules that can be implemented by ex post allocations. Our method can be used to characterize feasible interim allocations in general multi-unit auctions where agents face capacity constraints, both ceilings and floors. Applications include a variety of settings of practical interest, ranging from individual and group-specific capacity constraints, set-aside sale, partnership dissolution, and government license reallocation.
\end{abstract}

KEYwORDS: Reduced-form auctions, network-flow approach, feasible circulation flow, paramodular capacity constraints.

JEL-CODE: D44.

\section{INTRODUCTION}

In the classical auction design problem, a bidder's incentive constraint is used to express his payments in terms of interim allocations - his expected winning probabilities given his types. This allows one to express the seller's objective function solely in terms of interim allocation rules. Even though the standard approach due to Myerson (1981) has been to search pointwise for an ex post allocation that is optimal, one could instead solve for optimality in terms of an interim allocation rule.

Interim allocations rules are simpler objects than ex post allocations rules, because they are lower-dimensional functions. This can make a difference in computational and analytical tractability. Moreover, in some problems, the allocation rule cannot easily be optimized

Date: June 24, 2013.

We thank Dirk Bergemann, Awi Federgruen, Drew Fudenberg, Jacob Goeree, Johannes Hörner, Alexej Kushnir, Benny Moldovanu, Larry Samuelson, Jay Sethuraman, Rakesh Vohra, the editor Matt Jackson, three anonymous referees and audiences at Bonn, Boston College, Boston University, Columbia, PSE, UPenn, Stanford, Yale, the Game Theory World Congress 2012, Istanbul, ISMP Berlin 2012, KAIST, SUFE, and the 11th SSCW meeting, New Delhi for comments and discussions. Che and Kim acknowledge the support by WCU program through the National Research Foundation of Korea funded by the Ministry of Education, Science and Technology (R32-2008-000-10056-0). Mierendorff gratefully acknowledges financial support from the European Research Council (ERC Advanced Investigator Grant, ESEI-249433). Part of this research was conducted while Che visited the University of Zürich, Mierendorff visited Yonsei University, and Kim visited Columbia University; they are grateful for the hospitality of the hosting institutions.

Che: Department of Economics, Columbia University and YERI, Yonsei University, yeonkooche@gmail.com. Kim: Department of Economics, Seoul National University, jikim72@gmail.com.

Mierendorff (corresponding author): Chair of Organizational Design, Department of Economics, University of Zürich, konrad.mierendorff@econ.uzh.ch. 
point-wise for each type profile. For instance, agents may face constraints in their payments for a variety of reasons. ${ }^{1}$ Given the envelope condition, such payment constraints can be readily checked for an interim allocation rule, but not for an ex post allocation rule. A similar situation is encountered if agents have type-contingent outside options. ${ }^{2}$ Again, such constraints can be checked for agents' interim allocation rules (via the envelope expression), but not for ex post allocation rules. For these reasons and others, the interim approach, employed first by Maskin and Riley (1984), has become increasingly popular in mechanism design research. ${ }^{3}$

For this approach to work, however, one must characterize the set of interim allocation rules that are implementable in the sense that there exists an ex post allocation rule generating the desired interim winning probabilities. Implementable interim allocation rules are also called reduced form auctions. Proving a conjecture by Matthews (1984), Border (1991, 2007) characterized implementable interim allocation rules for the single-unit auction case. ${ }^{4}$ Because of its tractable structure, this characterization has has proven useful for mechanism design analysis, but its scope has been limited to one-unit auctions, and so far it has remained unclear whether a characterization can be obtained for a more general setting.

In this paper, we extend the characterization of reduced-form auctions to a general multiunit setting with bidder capacity constraints, while retaining the tractable structure for Border's original contribution. The novelty of our approach is to view implementation of an interim allocation rule as a problem of assigning desired (fractional) units of the good to different types of agents using the supply available at different ex post states (i.e., profiles of bidders' types). The solution to the assignment problem corresponds to a feasible flow in an appropriately defined network. We thus convert the problem of whether an interim allocation rule is implementable into the problem of whether there exists a feasible flow in a certain network. To derive our characterization of implementable interim allocation rules,

\footnotetext{
${ }^{1}$ For instance, the agents may be financially constrained (Che and Gale, 1998, 2000; Laffont and Robert, 1996; Maskin, 2000; Pai and Vohra, 2011). In the context of collusion, members of a cartel may refrain from using monetary transfers, for fear of detection (McAfee and McMillan, 1992; Che et al., 2012). Or monetary transfers may be simply unavailable for other reasons (Miralles, 2012; Che et al., 2013).

${ }^{2}$ See Mierendorff (2009).

${ }^{3}$ See for example Armstrong (2000), Asker and Cantillon (2010), Parlane (2001), Brusco and Lopomo (2002), Manelli and Vincent (2010), Hörner and Samuelson (2011), Miralles (2012), Pai and Vohra (2012), Pai (2012), and Che et al. (2012).

${ }^{4}$ For the case of asymmetric agents, Mierendorff (2011) and Che et al. (2012) offer a tighter characterization than Border (2007). Shi (2009) and Alaei et al. (2012) extend the characterization to allow for certain types of capacity constraints, which are special cases of our framework. Gershkov et al. (2013) point out that the analysis of reduced forms is related to the problem of finding a distribution with given marginals (see references in Gershkov et al., 2013). This leads to a majorization condition that characterizes reduced forms (see Gale, 1957). Gale's construction, however, only works for two buyers. Also, his condition differs from Border's and seems less tractable. For a related characterization for symmetric allocation rules see Hart and Reny (2011). Goeree and Kushnir (2011) characterize reduced forms in terms of support functions of the feasible set.
} 
we construct such a network and invoke the conditions for existence of a feasible flow from the network-flow literature (see Hassin, 1982). ${ }^{5}$

For a single-unit auction, our characterization reduces to the one obtained in the existing literature. In this case, our method makes the insight of the feasibility condition transparent. More importantly, we provide a characterization of interim allocations in a general multi-unit environment in which subsets of agents face capacity constraints both in upper and lower bounds. These constraints are required to be paramodular, meaning that upper bounds are submodular, lower bounds are supermodular, and the two bounds are compliant-a property that ensures that no constraints are redundant. We show that all of these properties are necessary for a characterization that has a tractable structure. If capacity constraints are only imposed on a hierarchical family of sets, we show that paramodularity is fulfilled automatically. Under restrictions on the environment, such as stochastic independence of type distributions, and/or symmetry among a set of agents, our characterization reduces to a much smaller number of inequalities, which considerably enhances the tractability and applicability of the reduced-form auction method.

Our domain includes a number of practically important settings. For an individual agent, a capacity constraint may arise from his limited ability to utilize the units he obtains. For instance, firms can profitably utilize at most a finite number of units (e.g., spectrum licenses). Constraints on groups of agents may arise from the seller's (e.g., the government's) desire to nurture minority participation or to preserve a competitive (post-assignment) industry. For instance, the government may wish to limit the number of units accruing to large or incumbent firms, making the remaining units available for small firms or new entrants. Lower bounds arise if units are set aside for some designated (e.g., minority) group. Also, partnership dissolution problems and the reallocation of government licenses can be modeled using a lower bound on the total number of units to be allocated.

In Section 2, we present the general model with capacity constraints and provide the conditions that characterize reduced form auctions. In Section 3, we show how the constraints can be reduced if types are independent and if groups of bidders are ex-ante symmetric. In Section 4, we provide applications where capacity constraints are imposed on partitioned sets of agents. All proofs can be found in the Appendix and the Supplementary Material.

\section{Reduced-Form Auctions with Capacity Constraints}

2.1. Notation. Let $I=\{1, \ldots,|I|\}$ be the set of agents with typical elements $i, j \in I$. For each agent $i$, there is a finite set of types $\Theta_{i}$ with typical element $\theta_{i} \in \Theta_{i}$. We show in Section 2.5 that our results generalize for general type spaces. As usual, we define $\Theta:=\times_{i \in I} \Theta_{i}$ and

\footnotetext{
${ }^{5}$ Our use of network flow techniques differs from the analysis of incentive constraints using a network approach (see Vohra, 2011). While we study an assignment problem for which the existence of a feasible flow is central, the analysis of incentive constraints requires solving a shortest-path problem.
} 
$\Theta_{-i}:=\times_{j \neq i} \Theta_{j}$. For a type profile $\theta \in \Theta, p(\theta) \in[0,1]$ denotes the probability that this profile, or "ex-post state," is realized. The marginal distribution of types of any agent $i$ is denoted by $p_{i}\left(\theta_{i}\right)$ and we assume that $p_{i}\left(\theta_{i}\right)>0$ for all $i \in I$ and $\theta_{i} \in \Theta_{i}$. The probability of a type profile $\left(\theta_{i}, \theta_{-i}\right)$ conditional on $\theta_{i}$ is denoted by $p_{-i}\left(\theta_{-i} \mid \theta_{i}\right)=p\left(\theta_{i}, \theta_{-i}\right) / p_{i}\left(\theta_{i}\right)$.

It will be convenient to work with the disjoint union of the individual type-spaces $D:=$ $\bigsqcup_{i \in I} \Theta_{i}$. The disjoint union differs from the standard union in that the elements are indexed by the set they come from. Formally, $D=\bigsqcup_{i \in I} \Theta_{i}=\bigcup_{i \in I}\left\{\left(\theta_{i}, i\right) \mid \theta_{i} \in \Theta_{i}\right\}$. To simplify notation we write typical elements of $D$ as $\theta_{i}$ instead of $\left(\theta_{i}, i\right)$. For example, if $I=\{1,2\}$ and $\Theta_{1}=\Theta_{2}=\{\underline{\theta}, \bar{\theta}\}$, we have $D=\left\{\underline{\theta}_{1}, \bar{\theta}_{1}, \underline{\theta}_{2}, \bar{\theta}_{2}\right\}$. Any subset $T \subset D$ can be written as the disjoint union $\bigsqcup_{i \in I} T_{i}$ of subsets $T_{i} \subset \Theta_{i}$. In the example, $T=\left\{\underline{\theta}_{1}, \bar{\theta}_{1}, \underline{\theta}_{2}\right\}=T_{1} \sqcup T_{2}$, where $T_{1}=\{\underline{\theta}, \bar{\theta}\}$ and $T_{2}=\{\underline{\theta}\}$.

2.2. Allocation rules and constraints. There are $n$ units of a good to be allocated. We assume that any subset $G \subset I$ of agents can receive at most $C(G)$ units and must receive at least $L(G)$ units of the good. Formally we define two mappings, $C: 2^{I} \rightarrow \mathbb{R}_{+}$and $L: 2^{I} \rightarrow \mathbb{R}_{+}$, with $C(\emptyset)=L(\emptyset)=0$. Without loss of generality we can take $C(I)=n$.

We say that an (ex-post) allocation rule $q: \Theta \rightarrow[0, n]^{|I|}$ respects $(C, L)$, if

$$
\forall G \subset I, \forall \theta \in \Theta: \quad L(G) \leq \sum_{i \in G} q_{i}(\theta) \leq C(G){ }^{6}
$$

A given ex post allocation rule $q$ induces an interim allocation rule $Q=\left(Q_{1}, \ldots, Q_{|I|}\right)$, where $Q_{i}: \Theta_{i} \rightarrow[0, n]$ represents $i$ 's expected assignment given his type. For each $i \in I$ and $\theta_{i} \in \Theta_{i}$, we have

$$
Q_{i}\left(\theta_{i}\right):=\sum_{\theta_{-i} \in \Theta_{-i}} q_{i}\left(\theta_{i}, \theta_{-i}\right) p\left(\theta_{-i} \mid \theta_{i}\right) .
$$

Conversely, one could begin with an arbitrary interim allocation rule and ask whether it can be implemented by an ex post allocation rule. As motivated in the introduction, such an approach is necessary in certain situations. Formally, an interim allocation rule $\left(Q_{i}: \Theta_{i} \rightarrow[0, n]\right)_{i \in I}$ is implementable if it is the reduced form of an ex-post allocation rule, i.e., if there exists an ex-post allocation rule $q$ satisfying (1) and (2).

Throughout the paper, we make the assumption that the constraints $(C, L)$ satisfy paramodularity: ${ }^{7}$

(1) $C$ is submodular: for any $G, G^{\prime} \subset I, C(G)+C\left(G^{\prime}\right) \geq C\left(G \cup G^{\prime}\right)+C\left(G \cap G^{\prime}\right)$.

(2) $L$ is supermodular: for any $G, G^{\prime} \subset I, L(G)+L\left(G^{\prime}\right) \leq L\left(G \cup G^{\prime}\right)+L\left(G \cap G^{\prime}\right)$.

(3) $C$ and $L$ are compliant: for any $G, G^{\prime} \subset I, C\left(G^{\prime}\right)-L(G) \geq C\left(G^{\prime} \backslash G\right)-L\left(G \backslash G^{\prime}\right)$.

\footnotetext{
${ }^{6}$ The associated set is known as a generalized polymatroid (see Schrijver (2000)).

${ }^{7}$ Notice that supermodularity implies that $L$ is non-decreasing and compliance implies that $C$ is nondecreasing.
} 
The assumption of submodularity makes the ceilings of larger groups of agents relevant. For instance, the ceiling for $I=\{1,2\}$ is only relevant if it does not exceed the sum of the individual quotas for agents 1 and 2 , as required by submodularity. ${ }^{8}$ Likewise, supermodularity of $L$ makes the floors of larger groups relevant. Finally, the compliance property captures consistency across alternative constraints, ensuring that the feasible set is non-empty, ${ }^{9}$ and that the upper bound and lower bounds are effective. ${ }^{10}$

The allocation of a single object is a special case of paramodular constraints with $C(G)=1$ for $G \neq \emptyset$ and $L(G) \equiv 0$. Another example is that the maximum capacity for a group simply depends on the number of agents in the group $C(G)=\psi(|G|)$. If there are no lower bounds $(L(G) \equiv 0)$, and $\psi$ is weakly concave, the resulting constraints are paramodular.

But our framework encompasses a much broader range of scenarios. One important case is that capacity constraints are imposed only on a hierarchical family of subsets. This structure is particularly useful since a hierarchical family gives rise to a paramodular constraint structure, regardless of the exact values of the ceilings and floors imposed for this family. To be specific, consider a family $\mathcal{H} \subset 2^{I} \backslash \emptyset$ of sets of agents which is hierarchical in that for any $G, G^{\prime} \in \mathcal{H}$ either $G \cap G^{\prime}=\emptyset, G \subset G^{\prime}$ or $G \supset G^{\prime}$. Each subset $G \in \mathcal{H}$ faces a ceiling denoted by $C_{G} \in[0, n]$ and a floor denoted by $L_{G} \in[0, n]$ and there are no constraints imposed directly on $G \notin \mathcal{H}$. Without loss, we assume that $I$ is included in $\mathcal{H}$. (If we add $I, \mathcal{H}$ remains hierarchical and setting $C_{I}=n$ and $L_{I}=0$ does not affect any constraints.) A hierarchical family $\mathcal{H}$ together with constraints $\left(G_{G}, L_{G}\right)_{G \in \mathcal{H}}$ defines a feasible set of allocations

$$
\mathcal{P}:=\left\{\left(x_{1}, \ldots, x_{|I|}\right) \in[0, n]^{|I|} \mid L_{G} \leq \sum_{i \in G} x_{i} \leq C_{G}, \forall G \in \mathcal{H}\right\} .
$$

This allows us to derive effective ceilings and floors for all subsets $G \subset I$. We define

$$
\forall G \subset I: \quad C(G):=\max \left\{\sum_{i \in G} x_{i} \mid\left(x_{1}, \ldots, x_{|I|}\right) \in \mathcal{P}\right\},
$$

\footnotetext{
${ }^{8}$ This is not to claim that the condition is without loss of generality. For example, submodularity fails when $C(\{1,2\})=C(\{2,3\})=C(\{2\})=1$ and $C(\{1,2,3\})=2$, although each of these ceilings can be binding.

${ }^{9}$ For instance, if $G=G^{\prime}$, the condition simplifies to $C(G) \geq L(G)$, which is clearly necessary for a feasible allocation to exist. Note that in contrast to Schrijver (2000), we require compliance also for sets $G \subset G^{\prime}$ and $G^{\prime} \subset G$. This rules out an unnecessary slack in the ceilings for certain sets.

${ }^{10}$ The effective upper bound for a set $G \subset I$ is given by $\max \left\{\sum_{i \in G} q_{i} \mid q\right.$ respects $\left.(C, L)\right\}$. Similarly, the effective lower bound for $G$ is given by $\min \left\{\sum_{i \in G} q_{i} \mid q\right.$ respects $\left.(C, L)\right\}$. In general, lower bounds interact with upper bounds in a way that may cause the effective bounds to differ from them. To illustrate, suppose $G^{\prime} \subset G$. Then, the set $G \backslash G^{\prime}$ cannot receive less than $L(G)-C\left(G^{\prime}\right)$, i.e., the difference between the minimum $G$ must receive and the maximum $G^{\prime}$ can receive. Hence, for $L\left(G^{\prime} \backslash G\right)$ to be effective, we must have $L\left(G \backslash G^{\prime}\right) \geq L(G)-C\left(G^{\prime}\right)$, which is precisely what compliance requires. Compliance is a sufficient condition for submodular upper bounds and supermodular lower bounds to be effective (Frank and Tardos, 1988), and there is a sense in which compliance constitutes a weakest sufficient condition or a maximal domain for the bounds to be effective. For details, see Section B of the Supplementary Material.
} 
with $C(\emptyset)=0$, and

$$
\forall G \subset I: \quad L(G):=\min \left\{\sum_{i \in G} x_{i} \mid\left(x_{1}, \ldots, x_{|I|}\right) \in \mathcal{P}\right\},
$$

with $L(\emptyset)=0$. The following Lemma shows that the ceilings and floors $(C(G), L(G))_{G \subset I}$ indeed describe the feasible set $\mathcal{P}$, and moreover, the constraints are paramodular regardless of the original constraints for the hierarchical family $\left(G_{G}, L_{G}\right)_{G \in \mathcal{H} \cdot}{ }^{11}$

Lemma 1. Consider any hierarchical family $\mathcal{H} \subset 2^{I} \backslash \emptyset$ that contains $I$ and arbitrary capacities $\left(C_{G}, L_{G}\right)_{G \in \mathcal{H}} \in[0, n]^{2|\mathcal{H}|}$. If the feasible set $\mathcal{P}$ is non-empty, then it is described by the effective constraints given by (3) and (4), that is,

$$
\mathcal{P}=\left\{\left(x_{1}, \ldots, x_{|I|}\right) \in \mathbb{R}_{+}^{|I|} \mid L(G) \leq \sum_{i \in G} x_{i} \leq C(G), \forall G \subset I\right\},
$$

and $(C(G), L(G))_{G \in I}$ satisfy paramodularity.

Capacity constraints for hierarchical sets of agents are relevant in auctions in which the government imposes a cap on the number of units allocated to each of non-overlapping groups of bidders, such as incumbents and entrants, or domestic and foreign firms. ${ }^{12}$ Consider for example the case that $I=\{1,2,3\}$, where buyer 1 and 2 are incumbents and buyer 3 is an entrant. If the seller wishes to limit the total number of units allocated to the incumbents and also to prevent each individual firm from obtaining too many units, constraints may be placed on the following groups: $\mathcal{H}=\{I,\{1,2\},\{1\},\{2\},\{3\}\}$. Suppose the seller has $n=C_{I}=7$ units available, limits the number of units allocated to each buyer $i$ at $C_{\{i\}}=3$ and to the incumbents at $C_{\{1,2\}}=5$, respectively. With these constraints, we have $C(G)=C_{G}$ for all $G \in \mathcal{H}$. For the remaining sets $\{1,3\}$ and $\{2,3\}$, the individual constraints are binding and we have $C(\{1,3\})=C(\{2,3\})=2 C_{\{i\}}=6$. Submodularity is easily checked in this example.

Lower bounds on the allocation are present, for instance, if some agents may be suppliers, instead of buyers, of the good. Lower bounds are also relevant in the partnership dissolution problem. Since all shares of the partnership are initially owned by the agents, we must have $L(I)=C(I)=1$. Similarly, the FCC's proposed spectrum repurposing program seeks to reallocate the frequencies held by TV broadcasting licensees to firms who can make a more productive use of them (e.g., mobile telephone companies), which again leads to

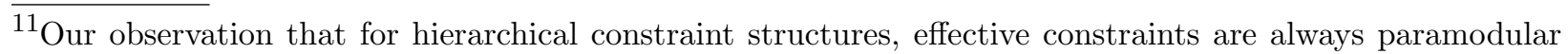
is related to the universal implementation results for random assignments with bi-hierarchical constraints in Budish et al. (2013). See Section C of the Supplementary Material.

${ }^{12}$ See Kim et al. (2012) for a procurement auction for school meals run by the Chilean government in which there are upper bounds on the number of units allocated to each participants. We thank an anonymous referee for pointing us to this paper.
} 


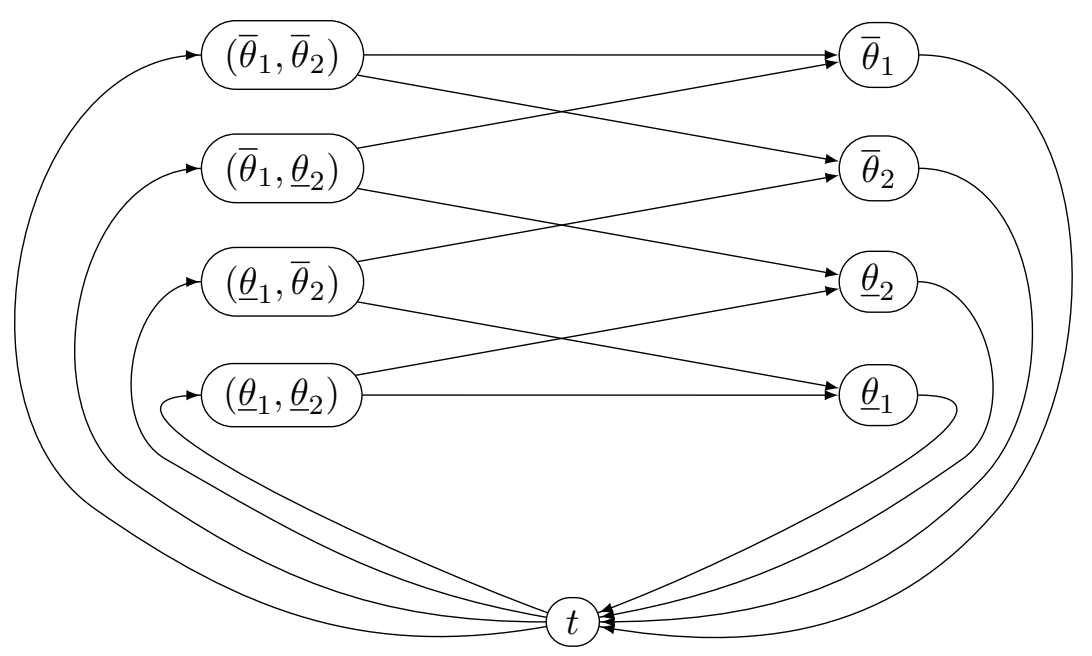

FiguRE 2.1. Illustration of the network.

$L(I)=n=C(I) \cdot{ }^{13}$ Of course, our model can also handle more general environments in which both new units and existing units are assigned. For instance, if $m$ units are currently owned by some agents and $n-m$ units are newly allocated, then we have $L(I)=m<n=C(I)$. Finally, lower bound constraints are also relevant when a government sets aside some units for a certain group of buyers, such as small business and minority. If the government guarantees the group to receive at least $k$ units, then $L(G)=k{ }^{14}$ In Section 4, we show how our characterization simplifies in these cases.

2.3. Network Flow Formulation. Before describing our network-flow framework formally, we explain its usefulness in a simple $2 \times 2$ example with i.i.d. types.

Example 1. There are two buyers $I=\{1,2\}$. Each buyer has two possible types, $\Theta_{i}=\{\bar{\theta}, \underline{\theta}\}$. Types are independently and identically distributed and both types are equally likely.

Figure 2.1 depicts the resulting network. The middle part of the network (without node t) consists of four nodes on the left representing the alternative type profiles or "ex post states" $\Theta=\left\{\left(\bar{\theta}_{1}, \bar{\theta}_{2}\right),\left(\bar{\theta}_{1}, \underline{\theta}_{2}\right),\left(\underline{\theta}_{1}, \bar{\theta}_{2}\right),\left(\underline{\theta}_{1}, \underline{\theta}_{2}\right)\right\}$, and four nodes on the right representing agents' types, or "interim states" $D=\bigsqcup_{i \in I} \Theta_{i}=\left\{\underline{\theta}_{1}, \bar{\theta}_{1}, \underline{\theta}_{2}, \bar{\theta}_{2}\right\}$. Our main insight is that the implementation of a given interim allocation rule can be seen as a problem of assigning scarce capacities available in each ex post state to "compatible" interim states. To be concrete, consider the interim allocation rule $\left(Q_{1}\left(\bar{\theta}_{1}\right), Q_{1}\left(\underline{\theta}_{1}\right), Q_{2}\left(\bar{\theta}_{2}\right), Q_{2}\left(\underline{\theta}_{2}\right)\right)=(1.5,2,2,1)$. This interim allocation rule requires, for instance, that agent 1 must receive on average 1.5 units

\footnotetext{
${ }^{13}$ See Notice of Proposed Rulemaking by FCC 12-118 (October 2, 2012), which spells out the plan to purchase licenses of $600 \mathrm{MHz}$ frequencies from TV broadcast stations through a reverse auction and reallocate them to mobile companies through a forward auction, and use the sale proceeds from the latter to finance the purchase in the former auction.

${ }^{14}$ This policy differs from capping the complementary group $I \backslash G$ to at most $n-k$ units. With a cap on $I \backslash G$ some of the units set aside for $G$ may remain unassigned.
} 
when his type is $\bar{\theta}_{1}$. But an assignment to $\bar{\theta}_{1}$ is only possible in "compatible" states - namely those states $\left(\theta_{1}, \theta_{2}\right)$ where agent 1 has type $\theta_{1}=\bar{\theta}_{1}$. Hence, the average assignment in states $\left(\bar{\theta}_{1}, \bar{\theta}_{2}\right)$ and $\left(\bar{\theta}_{1}, \underline{\theta}_{2}\right)$ to $\bar{\theta}_{1}$ must be 1.5 units.

The problem of assigning scarce resources on one side of the market to meet the demands of the other side is known as Hall's marriage problem. In the marriage problem, there are men on one side and women on the other, and each man is compatible with a subset of women. The question is then whether all of the agents on one side, say men, can be fully matched with compatible agents, women, on the other side. Our problem can be seen as a marriage problem: the (ex ante) quantity to be implemented for interim state $\theta_{i}, Q_{i}\left(\theta_{i}\right) p_{i}\left(\theta_{i}\right)$ can be interpreted as the number of "men" with type $\theta_{i}$, and the (ex ante) capacity available at ex post state $\left(\tilde{\theta}_{1}, \tilde{\theta}_{2}\right), C(I) p\left(\tilde{\theta}_{1}, \tilde{\theta}_{2}\right)$, can be interpreted as the number of women with type $\left(\tilde{\theta}_{1}, \tilde{\theta}_{2}\right)$. Men with type $\theta_{i}$ are only compatible with women whose type $\left(\tilde{\theta}_{1}, \tilde{\theta}_{2}\right)$ satisfies $\tilde{\theta}_{i}=\theta_{i}$. Described in this way, the implementability question can simply be recast as the question whether all men can be matched with compatible women. Hall's marriage theorem provides necessary and sufficient conditions for the existence of an integer matching under very simple capacity constraints. ${ }^{15}$ The network flow approach we adopt generalizes Hall's characterization in a fractional assignment setting with a much more general class of constraints.

In order to characterize the set of implementable interim allocation rules for a given constraint structure $(C, L)$, we define a circulation network $(N, E, k, d)$ which consists of nodes $N$, edges $E$, and a pair of functions $(k, d)$ that specify ceilings and floors for the flow on all subsets of outgoing edges for each node.

Nodes: The node set $N=D \cup \Theta \cup\{t\}$ consists of demand nodes $D$, supply nodes $\Theta$ and a circulation node $t$.

Edges: Directed edges $E \subset N \times N$ specify the pairs of nodes which can carry flows. There are three different kinds edges:

- Edges from supply nodes to demand nodes: We specify a directed edge from an ex post state $\tilde{\theta}=\left(\tilde{\theta}_{1}, \ldots, \tilde{\theta}_{|I|}\right) \in \Theta$ to an interim state $\theta_{i} \in D$ if and only if they are "compatible" in the sense that $\theta_{i}=\tilde{\theta}_{i}$. In the example, agent one with type $\bar{\theta}_{1}$ can only receive supply in ex-post states $\left(\bar{\theta}_{1}, \bar{\theta}_{2}\right)$ and $\left(\bar{\theta}_{1}, \underline{\theta}_{2}\right)$.

- Edges from demand nodes to the circulation node $t$ : We specify an edge $\left(\theta_{i}, t\right)$ from each demand node $\theta_{i} \in D$ to $t$.

- Edges from the circulation node to the supply nodes: We specify an edge $(t, \theta)$ from $t$ to each supply node $\theta \in \Theta$.

\footnotetext{
${ }^{15}$ Hall's characterization states that all men can matched with compatible women if and only if, for any subset of men, the number of women compatible with at least one of them is no less than the number of men in that subset. This has the flavor of Border's characterization, as will become clearer.
} 
Flow capacities: We specify upper and lower bounds on outgoing flows for each node in the network. Formally, for each node $n \in N$ and each subset $N^{\prime} \subset N \backslash\{n\}$ of the remaining nodes, we let $d\left(n, N^{\prime}\right)$ and $k\left(n, N^{\prime}\right)$ denote a lower and upper bound for the (total) flow from $n$ to $N^{\prime}$, respectively. If there are no edges from $n$ to $N^{\prime}$, we will of course define $k\left(n, N^{\prime}\right)=d\left(n, N^{\prime}\right)=0$.

To define the capacities, we introduce the following notation. For any ex post state $\theta=\left(\theta_{1}, \ldots, \theta_{|I|}\right) \in \Theta$ and a set $D^{\prime} \subset D$ of interim states, let $I\left(\theta, D^{\prime}\right):=\left\{i \in I \mid \theta_{i} \in D^{\prime}\right\}$ denote the set of agents who have types in $D^{\prime}$ that are compatible with $\theta$. In other words, the set $I\left(\theta, D^{\prime}\right)$ consists of the agents who can receive the good at state $\theta$, if allocations are restricted to interim types in $D^{\prime}{ }^{16}$

- Flow capacities from supply nodes: These constraints reflect the constraints on the ex-post assignment as defined by $(C, L)$. For each supply node $\theta \in \Theta$, we define

$$
k\left(\theta, N^{\prime}\right)=p(\theta) C\left(I\left(\theta, N^{\prime} \cap D\right)\right) \quad \text { and } \quad d\left(\theta, N^{\prime}\right)=p(\theta) L\left(I\left(\theta, N^{\prime} \cap D\right)\right) .
$$

Note that $k\left(\theta, N^{\prime}\right)=d\left(\theta, N^{\prime}\right)=0$ if there is no directed edge $\left(\theta, n^{\prime}\right) \in E$ for some node $n^{\prime} \in N^{\prime}$. If there are directed edges from $\theta$ to some nodes in $N^{\prime}$, these edges point to demand nodes in $D^{\prime}=N^{\prime} \cap D$. We cap the flow from $\theta$ to demand nodes $D^{\prime}$ by the maximal number of units that the set $I\left(\theta, D^{\prime}\right)$ of agents can receive and floor it by the minimal number of units that the same set of agents must receive, in ex ante terms (i.e., multiplied by the probability $p(\theta)) .{ }^{17}$

- Flow capacities from demand nodes: These constraints reflect the quantities that each interim type must be assigned in order to implement a given interim allocation rule $Q$. For each demand node $\theta_{i} \in D$, we let $k\left(\theta_{i}, N^{\prime}\right)=d\left(\theta_{i}, N^{\prime}\right)=p_{i}\left(\theta_{i}\right) Q_{i}\left(\theta_{i}\right)$ if $t \in N^{\prime}$, or else $k\left(\theta_{i}, N^{\prime}\right)=d\left(\theta_{i}, N^{\prime}\right)=0$. In words, we are "forcing" the outgoing flow at each demand node $\theta_{i}$ to match exactly the desired level of interim quantity $p_{i}\left(\theta_{i}\right) Q_{i}\left(\theta_{i}\right)$ in ex ante terms (i.e., multiplied by the marginal probability $\left.p_{i}\left(\theta_{i}\right)\right)$.

- Flow capacities from $t$ : Finally, we set $d\left(t, N^{\prime}\right)=0$ and $k\left(t, N^{\prime}\right)=K$, where $K>0$ is a sufficiently large number. This is to ensure that constraints on the edges emanating from $t$ never bind.

Feasible circulation flow. A feasible circulation flow on $(N, E, k, d)$ is a function $f$ : $E \rightarrow \mathbb{R}_{+}$that satisfies the capacity constraints,

$$
d\left(n, N^{\prime}\right) \leq \sum_{n^{\prime} \in N^{\prime}:\left(n, n^{\prime}\right) \in E} f\left(n, n^{\prime}\right) \leq k\left(n, N^{\prime}\right), \quad \forall n \in N, \forall N^{\prime} \subset N \backslash\{n\},
$$

\footnotetext{
${ }^{16}$ For instance, consider state $\left(\bar{\theta}_{1}, \underline{\theta}_{2}\right)$ in the example and let $D^{\prime}=\left\{\bar{\theta}_{1}, \bar{\theta}_{2}\right\}$. Only agent 1 's type in the state $\left(\bar{\theta}_{1}, \underline{\theta}_{2}\right)$ is contained in $D^{\prime}$ so $I\left(\left(\bar{\theta}_{1}, \underline{\theta}_{2}\right),\left\{\bar{\theta}_{1}, \bar{\theta}_{2}\right\}\right)=\{1\}$.

${ }^{17}$ In our example, $k\left(\left(\bar{\theta}_{1}, \bar{\theta}_{2}\right),\left\{\bar{\theta}_{1}\right\}\right)=p\left(\bar{\theta}_{1}, \bar{\theta}_{2}\right) C(\{1\})$ is the capacity of the single edge from $\left(\bar{\theta}_{1}, \underline{\theta}_{2}\right)$ to $\bar{\theta}_{1}$. $k\left(\left(\bar{\theta}_{1}, \underline{\theta}_{2}\right),\left\{\bar{\theta}_{1}, \underline{\theta}_{2}\right\}\right)=p\left(\bar{\theta}_{1}, \underline{\theta}_{2}\right) C(\{1,2\})$ is the maximal flow that the edges $\left(\left(\bar{\theta}_{1}, \underline{\theta}_{2}\right), \bar{\theta}_{1}\right)$ and $\left(\left(\bar{\theta}_{1}, \underline{\theta}_{2}\right), \underline{\theta}_{2}\right)$ are allowed to carry in total.
} 
and the flow conservation law:

$$
\sum_{n^{\prime} \in N:\left(n, n^{\prime}\right) \in E} f\left(n, n^{\prime}\right)=\sum_{n^{\prime} \in N:\left(n^{\prime}, n\right) \in E} f\left(n^{\prime}, n\right), \quad \forall n \in N .
$$

In words, a circulation flow is feasible if the outgoing flows obey the capacity constraints given by $(k, d)$ and the total flow entering each node equals the total flow exiting it.

It is easy to see the implication of a feasible circulation flow on the implementability of an interim allocation rule. We can recover an ex-post allocation rule from a feasible flow on the network defined for a given interim allocation rule $Q$ :

$$
q_{i}(\theta)=\frac{f\left(\theta, \theta_{i}\right)}{p(\theta)} .
$$

Given our construction, any feasible flow must have

$$
f\left(\theta_{i}, t\right)=p_{i}\left(\theta_{i}\right) Q_{i}\left(\theta_{i}\right)
$$

Using these identities and flow conservation at $n=\theta_{i}$, we observe that

$$
p_{i}\left(\theta_{i}\right) Q_{i}\left(\theta_{i}\right)=f\left(\theta_{i}, t\right)=\sum_{\theta_{-i} \in \Theta_{-i}} f\left(\left(\theta_{i}, \theta_{-i}\right), \theta_{i}\right)=\sum_{\theta_{-i} \in \Theta_{-i}} p\left(\theta_{i}, \theta_{-i}\right) q_{i}\left(\theta_{i}, \theta_{-i}\right) .
$$

This shows that the allocation rule $q$ satisfies (2) and implements the reduced form $Q$. Conversely, implementability of a given reduced form implies the existence of a feasible flow:

Theorem 1. An interim allocation $Q$ is implementable if and only if there exists a feasible circulation flow for the network $(N, E, k, d)$ defined above.

We are now ready to invoke the following result from Hassin (1982): ${ }^{18}$

Theorem 2 (Hassin, 1982). For every $n \in N$, let $k(n, \cdot)$ and $d(n, \cdot)$ (defined on subsets of $N \backslash\{n\}$ ) be paramodular. Then, a feasible circulation flow $f: E \rightarrow \mathbb{R}_{+}$exists if and only if

$$
\sum_{n \in N \backslash M} d(n, M) \leq \sum_{n \in M} k(n, N \backslash M), \quad \forall M \subset N .
$$

Since $(C, L)$ are paramodular, it is straightforward to verify that the functions $k(n,$.$) and$ $d(n,$.$) in our network are paramodular for all nodes n \in N$. Condition (6) requires that the sum of lower bounds on the flows entering $M$ does not exceed the sum of upper bounds on the flows exiting $M$. Intuitively, this is necessary for the existence of a feasible circulation flow. Theorem 2 says that (6) is also sufficient.

2.4. Characterization of reduced form allocation rules. We now establish our characterization result by applying Theorem 2 to the network defined above. To this end, we

\footnotetext{
${ }^{18}$ Without floor constraints, we could employ a characterization of polymatroidal network flows due to Federgruen and Groenevelt (1988). We thank Rakesh Vohra and an anonymous referee for pointing us to that article.
} 
introduce the following notation. For any subset of demand nodes $T=\bigsqcup_{i \in I} T_{i} \subset D$, we define $Y(T):=\{\theta \in \Theta \mid I(\theta, T) \neq \emptyset\}$ to be the set of supply nodes that are "compatible" with $T$. These are the states at which at least one agent $i$ with type in $T_{i}$ can receive the good, i.e., $Y(T)=\bigcup_{i \in I}\left(T_{i} \times \Theta_{-i}\right) .{ }^{19}$

Theorem 3. Let $Q=\left(Q_{i}\right)_{i \in I}$ be an interim allocation rule. Then, $Q$ is the reduced form of an allocation rule that respects $(C, L)$ if and only if for all $T \subset D$,

$$
\sum_{\theta \in Y(T)} p(\theta) L(I(\theta, T)) \leq \sum_{i \in I} \sum_{\theta_{i} \in T_{i}} p_{i}\left(\theta_{i}\right) Q_{i}\left(\theta_{i}\right) \leq \sum_{\theta \in Y(T)} p(\theta) C(I(\theta, T)) .
$$

To understand the main argument of the proof, consider our $2 \times 2$ example and set $T=$ $\left\{\bar{\theta}_{1}, \bar{\theta}_{2}\right\}$. If we set $M=T$, (6) yields

$$
L(I) p\left(\bar{\theta}_{1}, \bar{\theta}_{2}\right)+L(\{1\}) p\left(\bar{\theta}_{1}, \underline{\theta}_{2}\right)+L(\{2\}) p\left(\underline{\theta}_{1}, \bar{\theta}_{2}\right) \leq p_{1}\left(\bar{\theta}_{1}\right) Q_{1}\left(\bar{\theta}_{1}\right)+p_{2}\left(\bar{\theta}_{1}\right) Q_{2}\left(\bar{\theta}_{2}\right)
$$

This is the left inequality in $\left(B^{\prime}\right)$ for $T=\left\{\bar{\theta}_{1}, \bar{\theta}_{2}\right\}$. Similarly, if we set $M=N \backslash T$, (6) yields the right inequality in $\left(B^{\prime}\right)$ for $T=\left\{\bar{\theta}_{1}, \bar{\theta}_{2}\right\}$ :

$$
p_{1}\left(\bar{\theta}_{1}\right) Q_{1}\left(\bar{\theta}_{1}\right)+p_{2}\left(\bar{\theta}_{1}\right) Q_{2}\left(\bar{\theta}_{2}\right) \leq C(I) p\left(\bar{\theta}_{1}, \bar{\theta}_{2}\right)+C(\{1\}) p\left(\bar{\theta}_{1}, \underline{\theta}_{2}\right)+C(\{2\}) p\left(\underline{\theta}_{1}, \bar{\theta}_{2}\right) .
$$

The proof is completed by showing that if (6) holds for $M=T$ and $M=N \backslash T$ for each $T \subset D$, then (6) is satisfied for all $M$, not just those corresponding to some $T \subset D$.

Conditions (7) and (8) bear resemblance to the reduced-form characterization familiar from the literature (see Border $(1991,2007)$ ), but there are notable differences. First of all, (7) has no analogue in the classical setting without lower bounds.

The second inequality deals with the upper bound, as in the existing literature, and simplifies to the familiar characterization if the agents face no capacity constraints. In that case, $C(I)=C(\{1\})=C(\{2\})=n$. The right-hand side of $(8)$ reduces to $n\left(p\left(\bar{\theta}_{1}, \bar{\theta}_{2}\right)+p\left(\bar{\theta}_{1}, \underline{\theta}_{2}\right)+\right.$ $\left.p\left(\underline{\theta}_{1}, \bar{\theta}_{2}\right)\right)=n\left(1-p\left(\underline{\theta}_{1}, \underline{\theta}_{2}\right)\right)$, i.e., the number of units multiplied by the probability that at least one agent has a type in $T$. Indeed, for the standard one-unit auction, where $C(G)=1$ for all non-empty $G \subset I$, and $L(G)=0$ for all $G \subset I$, our characterization simplifies to the familiar condition from Border (1991, 2007):

Corollary 1 (Border $(1991,2007))$. In the standard one-unit auction model, an interim allocation rule $q$ is the reduced form of an allocation rule if and only if for all $T \subset D$,

$$
\sum_{i \in I} \sum_{\theta_{i} \in T_{i}} Q_{i}\left(\theta_{i}\right) p_{i}\left(\theta_{i}\right) \leq \sum_{\theta \in Y(T)} p(\theta)
$$

Our characterization departs from the familiar condition when there are nontrivial capacity constraints on subsets $G \neq I$. For instance, suppose each agent faces a binding individual

$\overline{{ }^{19} \text { Recall that }} I(\theta, T)=\left\{i \in I \mid \theta_{i} \in T_{i}\right\}$ is the set of all agents with types in $T$ who can receive the good at state $\theta$. 


\begin{tabular}{ccccccccc}
\hline \hline$\psi(1)$ & $\psi(2)$ & $\psi(3)$ & $\phi(2)$ & $\phi(3)$ & violates & $\max$ & subject to & value \\
\hline 3 & 4 & 6 & 0 & 0 & subm. & $\sum_{i=1}^{3}\left\{Q_{i}(\underline{\theta})+2 Q_{i}(\bar{\theta})\right\}$ & $\begin{array}{c}\left(B^{\prime}\right) \\
(1) \&(2)\end{array}$ & $\begin{array}{c}18.375 \\
18\end{array}$ \\
\hline 2 & 4 & 6 & 2 & 3 & superm. & $-\sum_{i=1}^{3}\left\{Q_{i}(\underline{\theta})+2 Q_{i}(\bar{\theta})\right\}$ & $\begin{array}{c}\left(B^{\prime}\right) \\
(1) \&(2)\end{array}$ & $\begin{array}{c}-8.625 \\
-9\end{array}$ \\
\hline 2 & 3 & 4 & $6 / 5$ & 3 & compl. & $Q_{1}(\underline{\theta})-Q_{2}(\bar{\theta})$ & $\begin{array}{c}\left(B^{\prime}\right) \\
(1) \&(2)\end{array}$ & $\begin{array}{c}2 \\
1.9\end{array}$ \\
\hline \hline
\end{tabular}

TABLE I. Examples of effective constraints that violate paramodularity. $(\phi(1)=0$ in all examples. $)$

capacity constraint, i.e., $C(\{i\})<C(I)=n, i=1$, 2. In that case, the set $I(\theta, T)$ of agents who can receive the good at a given state $\theta$ when types are in $T$ matters. In particular, the characterization gives rise to a tighter condition than the standard one, and a failure to recognize this could lead to an interim allocation rule that is not implementable. ${ }^{20}$

Remark 1. Paramodularity of the capacity constraints is necessary for our characterization. To see this, suppose that there are three bidders $I=\{1,2,3\}$, each independently and equally likely to be of type $\underline{\theta}$ or $\bar{\theta}$. Each set of $k$ bidders faces an upper bound of $\psi(k)$ and a lower bound of $\phi(k)$. We give three examples (see Table I). For each example, the constraints are effective and exactly one of the conditions of paramodularity is violated while the others are satisfied. To demonstrate that the characterization is not valid in these examples, we first maximize a linear function in $Q$ subject to $\left(B^{\prime}\right)$. We compare the result to the maximum of the same objective function subject to the constraints (1) and (2). ${ }^{21}$ As the last column of Table I shows, the value is always strictly higher for maximization subject to $\left(B^{\prime}\right)$ in these examples. Therefore $\left(B^{\prime}\right)$ does not describe the set of reduced forms in any of these examples. $^{22}$

$\overline{{ }^{20} \text { To illustrate, }}$ suppose $p_{1}\left(\bar{\theta}_{1}\right)=p_{2}\left(\bar{\theta}_{2}\right)=p>2 / 3, C(I)=n=3$, and $C(\{1\})=C(\{2\})=2$. Consider the interim allocations given by $Q_{1}\left(\bar{\theta}_{1}\right)=Q_{2}\left(\bar{\theta}_{2}\right)=: \bar{Q}=3-(3 / 2) p$ and $Q_{1}\left(\underline{\theta}_{1}\right)=Q_{2}\left(\underline{\theta}_{2}\right)=\underline{Q}=(3 / 2)(1-p)^{2}$. It is straightforward to check that these interim allocations satisfy the standard Border constraints (e.g., Border (1991)). Yet, there is no allocation rule that implements these allocations and satisfies the additional constraint $q_{i}(\theta) \leq 2$. To see this, note that $q_{i}\left(\bar{\theta}_{1}, \bar{\theta}_{2}\right) \leq 3 / 2$ for at least one $i$, which follows from $q_{1}\left(\bar{\theta}_{1}, \bar{\theta}_{2}\right)+$ $q_{2}\left(\bar{\theta}_{1}, \bar{\theta}_{2}\right) \leq 3$. Using this, and $q_{i}\left(\bar{\theta}_{i}, \underline{\theta}_{-i}\right) \leq 2$, we have

$$
\bar{Q}=p q_{i}\left(\bar{\theta}_{i}, \bar{\theta}_{-i}\right)+(1-p) q_{i}\left(\bar{\theta}_{i}, \underline{\theta}_{-i}\right) \leq \frac{3}{2} p+2(1-p)=2-\frac{1}{2} p<3-\frac{3}{2} p,
$$

which is a contradiction.

${ }^{21}$ Details and a Mathematica file are available on request.

${ }^{22}$ For a model without lower bounds, but with general, possibly non-submodular, upper bounds, Cai et al. (2011) derive a characterization that involves a continuum of constraints. Instead of imposing a constraint for each subset of interim types $T \subset D$, i.e., a finite number of constraints as in our characterization, Cai et al. (2011) attach a weight $W_{i}\left(\theta_{i}\right) \in[0,1]$ to each interim type and impose a constraint for each profile of weights $\left(W_{i}\left(\theta_{i}\right)\right)_{i \in I, \theta_{i} \in \Theta_{i}}$. In Section D of the Supplementary Material, we show that, given submodular upper bounds, only constraints for integer weights have to be imposed, which implies that the characterization 
Remark 2. Linear programming problems subject to submodular upper bound constraints are known to be particularly tractable since a greedy algorithm can find an optimal solution (see Edmonds (1970)). An interesting observation by Vohra (2011) is that the optimal auction design problem has this special structure when formulated in reduced form and if the monotonicity constraint is neglected, since the Border characterization involves submodular upper bound constraints (i.e., the RHS of (9) is submodular in $T \subset D$ ). This observation can be extended to our more general multi-unit auctions with paramodular constraints: defining the RHS and LHS of $\left(B^{\prime}\right)$,respectively, as

$$
\Psi(T):=\sum_{\theta \in Y(T)} C(I(\theta, T)) p(\theta) \text { and } \Phi(T):=\sum_{\theta \in Y(T)} L(I(\theta, T)) p(\theta),
$$

we can establish the following result, whose proof is provided in Section A.1 of the Supplementary Material.

Theorem 4. $\Psi$ and $\Phi$ are paramodular.

Theorem 5 of Hassin (1982) shows that a "greedy-generous" algorithm can be used to solve linear optimization problems with paramodular constraints (i.e., including both upper and lower bounds). Given the above theorem, this means that an optimal auction design problem in our more general environment can be solved as tractably in reduced form as in the simple single-unit auction problem.

2.5. General Type Spaces. The characterization results in this and the following sections generalize to the case of general type distributions. Suppose that for each buyer $i \in I$, we have a probability space $\left(\Theta_{i}, \mathcal{A}_{i}, \mu_{i}\right)$, where $\Theta_{i}$ is the type-space, $\mathcal{A}_{i}$ is the $\sigma$-algebra of measurable sets, and $\mu_{i}$ is the marginal probability measure. The space of type profiles is given by $(\Theta, \mathcal{A}, \mu)$, where $\Theta=\Theta_{1} \times \ldots \times \Theta_{|I|}, \mathcal{A}$ is the product $\sigma$-algebra and $\mu$ is a probability measure on the product space with marginals $\mu_{i}$. The product spaces $\left(\Theta_{-i}, \mathcal{A}_{-i}, \mu_{-i}\right)$ are defined analogously.

An ex-post allocation rule that respects $(C, L)$ is a measurable function $q: \Theta \rightarrow[0, C(I)]^{|I|}$ that satisfies (2.1). An interim allocation rule is a measurable function $Q: \Theta \rightarrow[0, C(I)]^{|I|}$ such that $Q_{i}$ only depends on $\theta_{i}$. An interim allocation rule is implementable for given $(C, L)$ if there exists an ex-post allocation rule $q$, that respects $(C, L)$, such that $Q$ is the reduced form of $q$, i.e., $Q_{i}\left(\theta_{i}\right)=\int_{\Theta_{-i}} q_{i}\left(\theta_{i}, \theta_{-i}\right) d \mu_{-i}\left(\theta_{-i}\right)$.

Theorem 5. Let $Q: \Theta \rightarrow[0, C(I)]^{|I|}$ be measurable and $Q_{i}(\theta)=Q_{i}\left(\theta_{i}\right)$. $Q$ is the reduced form of an ex-post allocation rule that respects $(C, L)$ if and only if for all $T=\bigsqcup_{i \in I} T_{i} \subset D$, with $T_{i} \in \mathcal{A}_{i}$ for all $i \in I$,

of Cai et al. (2011) reduces to our characterization. Moreover, we demonstrate that for the first example in Table I, the maximizer subject to $\left(B^{\prime}\right)$ violates a constraint that corresponds to non-integer weights. Therefore, a tractable characterization that retains Border's original structure cannot be obtained without the assumption of paramodularity. 


$$
\int_{Y(T)} L(I(\theta, T)) d \mu(\theta) \leq \sum_{i \in I} \int_{T_{i}} Q_{i}\left(\theta_{i}\right) d \mu_{i}\left(\theta_{i}\right) \leq \int_{Y(T)} C(I(\theta, T)) d \mu(\theta) .
$$

The formal proof can be found in Section A.2 of the Supplementary Material.

\section{Reduction of Constraints}

The characterization in the previous section involves $2 \prod_{i \in I}\left|2^{\Theta_{i}}\right|$ inequalities. Since this number grows very quickly with the cardinalities of the type spaces, the condition is not very tractable. In this section, we derive two reductions that lead to more tractable characterizations. ${ }^{23}$ First, we show that if types are independently distributed, it is sufficient to check $\left(B^{\prime}\right)$ for the upper and lower contour sets of the interim allocation functions, i.e., sets of types whose interim allocations are no smaller and no larger, respectively, than certain thresholds. With this reduction, the number of inequalities that we need to check becomes much smaller, $2 \prod_{i \in I}\left|\Theta_{i}\right|$ at most. Second, we show that when some group(s) of agents are symmetric, and if we restrict attention to group-symmetric reduced forms, it suffices to check $\left(B^{\prime}\right)$ only for those $T$ for which the $T_{i}$ 's are identical for agents in the same group.

3.1. Independent Type Distribution. Consider the situation where agents' types are independently distributed, i.e., $p(\theta)=\prod_{i \in I} p_{i}\left(\theta_{i}\right), \forall \theta \in \Theta$. The following result shows that it is sufficient to check $\left(B^{\prime}\right)$ for upper and lower contour sets. ${ }^{24}$

Theorem 6. Suppose that the agents' types are independently distributed. Then, $Q$ is the reduced form of an allocation rule that respects $(C, L)$, if and only if

$$
\sum_{i \in I} \sum_{\theta_{i} \in T_{i}} p_{i}\left(\theta_{i}\right) Q_{i}\left(\theta_{i}\right) \leq \sum_{\theta \in Y(T)} p(\theta) C(I(\theta, T))
$$

${ }^{23}$ To our knowledge, all existing applications of reduced-form auctions mentioned in the introduction rely on the kind of "reduced" characterizations we provide in this section. When optimizing a linear objective, the greedy-generous algorithm discussed in Remark 2 can deal with the intractability by checking only one constraint in $\left(B^{\prime}\right)$ at each step. This, however, requires linearity and does not provide a characterization of implementable interim allocation rules. The reductions derived in this section, on the other hand, can be used when maximizing non-linear objective functions, or when other constraints are imposed in the maximization problem that invalidate a greedy-generous approach. The reductions are also useful to check the implementability of arbitrary interim allocation rules that are not necessarily extreme points of the feasible set.

${ }^{24}$ For correlated types, the constraints for upper and lower contour sets are generally not sufficient, as the following example demonstrates. Consider the standard one-unit auction without additional constraints for our $2 \times 2$ example with two buyers and two types, i.e., let $C(G) \equiv 1$ and $L(G) \equiv 0$. Let the type distribution be given by $p\left(\underline{\theta}_{1}, \underline{\theta}_{2}\right)=\frac{1}{10}, p\left(\underline{\theta}_{1}, \bar{\theta}_{2}\right)=\frac{1}{2}, p\left(\bar{\theta}_{1}, \underline{\theta}_{2}\right)=\frac{1}{5}, p\left(\bar{\theta}_{1}, \bar{\theta}_{2}\right)=\frac{1}{5}$. The interim allocation rule $Q_{1}\left(\underline{\theta}_{1}\right)=Q_{1}\left(\bar{\theta}_{1}\right)=0.27, Q_{2}\left(\underline{\theta}_{2}\right)=0.1, Q_{2}\left(\bar{\theta}_{2}\right)=1$ satisfies the upper contour set constraints. Nevertheless, the constraint for $T=\left(\left\{\underline{\theta}_{1}\right\},\left\{\bar{\theta}_{2}\right\}\right)$ is violated:

$$
\frac{3}{5} Q_{1}\left(\underline{\theta}_{1}\right)+\frac{7}{10} Q_{2}\left(\bar{\theta}_{2}\right)=\frac{3}{5} 0.27+\frac{7}{10}=.862>\frac{4}{5}=1-p\left(\bar{\theta}_{1}, \underline{\theta}_{2}\right) .
$$


for each $T=\bigsqcup_{i \in I} T_{i}$ with $T_{i}=\left\{\theta_{i} \in \Theta_{i} \mid Q_{i}\left(\theta_{i}\right) \geq e_{i}\right\}$ for some $e_{i} \geq 0$, and

$$
\sum_{\theta \in Y\left(T^{\prime}\right)} p(\theta) L\left(I\left(\theta, T^{\prime}\right)\right) \leq \sum_{i \in I} \sum_{\theta_{i} \in T_{i}^{\prime}} p_{i}\left(\theta_{i}\right) Q_{i}\left(\theta_{i}\right)
$$

for each $T^{\prime}=\bigsqcup_{i \in I} T_{i}^{\prime}$ with $T_{i}^{\prime}=\left\{\theta_{i} \in \Theta_{i} \mid Q_{i}\left(\theta_{i}\right) \leq e_{i}^{\prime}\right\}$ for some $e_{i}^{\prime} \geq 0$.

Bayesian incentive compatibility requires that interim allocations are monotonic, in which case the theorem entails even simpler conditions. With monotonicity, an upper contour set boils down to an interval of types above a threshold and a lower contour set reduces to an interval of types below a threshold. Hence, we obtain the following familiar characterization for single-unit auctions.

Corollary 2. Consider the standard single-unit setup (i.e., $C(G)=1$ for all nonempty $G \subset I$ and $L(G)=0$ for all $G$ ), and suppose that each $\Theta_{i}$ is linearly ordered and $q_{i}$ is nondecreasing. Then, $Q$ is the reduced form of an allocation rule if and only if for all $\left(\theta_{1}, \cdots, \theta_{|I|}\right) \in \Theta$

$$
\sum_{i \in N} \sum_{\theta_{i}^{\prime} \geq \theta_{i}} Q_{i}\left(\theta_{i}^{\prime}\right) p_{i}\left(\theta_{i}^{\prime}\right) \leq 1-\prod_{i \in I} P_{i}\left(\theta_{i}\right)
$$

where $P_{i}(\cdot)$ is the c.d.f. of $p_{i}(\cdot)$, i.e., $P_{i}\left(\theta_{i}\right)=\sum_{\theta_{i}^{\prime}<\theta_{i}} p_{i}\left(\theta_{i}^{\prime}\right)$.

3.2. Generalized Symmetric Environments. In many environments, there are sets of agents that share similar characteristics. For instance, in procurement auctions, the incumbents and entrants form two groups, and those within the same group have more in common in terms of technologies and other factors than those outside that group. In such a circumstance, it makes sense to view the agents within the same group as symmetric, and it often suffices to search for an optimal mechanism in the class of group-symmetric mechanisms, namely those that treat ex-ante identical buyers identically. As will be seen, with such mechanisms, the task of identifying reduced-forms can be reduced even further to checking $\left(B^{\prime}\right)$ only for group-symmetric sets $T$.

To be more specific, suppose that $I$ can be partitioned into subsets, $G_{1}, \ldots, G_{L}$. All agents in each non-singleton set (or group) $G_{\ell}$ are symmetric in the following sense: ${ }^{25}$ First, for all $i, j \in G_{\ell}, \Theta_{i}=\Theta_{j}=: \hat{\Theta}_{\ell}$. Second, $p$ is invariant to permutations of types for any pair of agents $i, j \in G_{\ell}$, i.e., $p\left(\theta_{i}, \theta_{j}, \theta_{-i j}\right)=p\left(\theta_{j}, \theta_{i}, \theta_{-i j}\right)$ for all $\theta_{i}, \theta_{j} \in \hat{\Theta}_{\ell}$ and all $\theta_{-i j} \in \Theta_{-i j}$. This implies that for each group, there exists a marginal distribution $\hat{p}_{\ell}: \hat{\Theta}_{\ell} \rightarrow[0,1]$, satisfying $p_{i}\left(\theta_{\ell}\right)=\hat{p}_{\ell}\left(\theta_{\ell}\right)$ for all $\theta_{\ell} \in \hat{\Theta}_{\ell}$ and all $i \in G_{\ell}$. Note that we do not require the type distribution to be independent. Third, while we allow for general paramodular capacity constraints, the capacity constraints involving any two agents from the same group must be identical, i.e., for any $i, j \in G_{\ell}, C\left(I^{\prime} \cup\{i\}\right)=C\left(I^{\prime} \cup\{j\}\right)$ and $L\left(I^{\prime} \cup\{i\}\right)=L\left(I^{\prime} \cup\{j\}\right)$ for all $I^{\prime} \subset I \backslash\{i, j\}$.

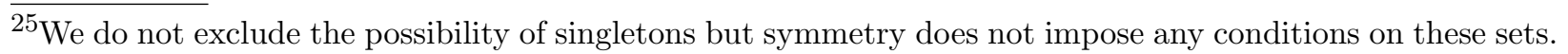


We call the environment described so far a generalized symmetric environment and establish a reduction of our characterization that applies to group-symmetric reduced forms. Formally, a reduced form is group-symmetric if for each group $G_{\ell}$, there exists an interim allocation rule $\hat{Q}_{\ell}: \hat{\Theta}_{\ell} \rightarrow \mathbb{R}_{+}$such that $Q_{i}\left(\theta_{\ell}\right)=\hat{Q}_{\ell}\left(\theta_{\ell}\right)$ for all $i \in G_{\ell}$ and all $\theta_{\ell} \in \hat{\Theta}_{\ell}$.

Theorem 7. In the generalized symmetric environment, a group-symmetric interim allocation rule $Q$ is a reduced form of an allocation rule that respects $(C, L)$ if and only if $\left(B^{\prime}\right)$ holds for all $T=\bigsqcup_{i \in I} T_{i}$ satisfying $T_{i}=T_{j}$ for all $i, j \in G_{\ell}$ and all $\ell=1, \ldots, L$.

If types are independently distributed, the reductions in Theorems 6 and 7 can be combined:

Corollary 3. Suppose the agents' types are independently distributed. Then, in the generalized symmetric environment, $Q$ is a reduced form of an allocation rule that respects $(C, L)$ if and only if $(B U)$ holds for all group-symmetric $T=\bigsqcup_{i \in I} T_{i}$ where each $T_{i}$ is an upper contour set of $q_{i}$ and $(B L)$ holds for all group-symmetric $T=\bigsqcup_{i \in I} T_{i}$ where each $T_{i}$ is a lower contour set of $q_{i}$.

The original characterization by Border (1991) and its extension by Mierendorff (2011) without capacity constraints (i.e. $C(G) \equiv 1$ and $L(G) \equiv 0$ ) are special cases of this corollary.

Remark 3. A group-symmetric interim allocation rule $Q$ satisfying the conditions of Theorem 4 may be implemented by an allocation rule $q$ that is not group-symmetric. Note, however, that we can uniformly randomize the identities of buyers that belong to the same group $G_{\ell}$ before applying the allocation rule $q$. We thereby construct a new allocation rule $\hat{q}$ that is group-symmetric and has the same reduced form, i.e., it also implements $q$.

\section{Applichtions: A Partitional Constraint Structure}

We now illustrate how our characterization can be applied to a variety of settings that are of interest to mechanism design. We do so by considering a partitional constraint structure. Suppose $n$ units of a good ("licenses") are allocated to a set $I$ of agents. A bidder $i$ values a unit of the good at $\theta_{i}$ distributed on a set $\Theta_{i} \subset \mathbb{R}_{+}$, with $\underline{\theta}_{i}:=\inf \Theta_{i}$ and $\bar{\theta}_{i}:=\sup \Theta_{i}$, according to a cumulative distribution function $F_{i}$. (The type distribution is either discrete or continuous.) Suppose the bidders are partitioned into different groups $\tilde{\mathcal{H}} \subset 2^{I}$, i.e., $\cup_{G \in \tilde{\mathcal{H}}} G=I$ and for all $G, G^{\prime} \in \tilde{\mathcal{H}}, G \cap G^{\prime}=\emptyset$. Each group $G \in \tilde{\mathcal{H}}$ faces an upper bound of $C_{G}$ and a lower bound of $L_{G}$, where $0 \leq L_{G} \leq C_{G} \leq n$ for all $G \in \tilde{\mathcal{H}}$. If we set $\mathcal{H}=\tilde{\mathcal{H}} \cup\{I\}$, we obtain a special case of the hierarchical structure introduced in Section 2.2. To make the constraints for $G=I$ effective, we assume that $\sum_{G \in \tilde{\mathcal{H}}} C_{G} \geq C_{I} \geq L_{I} \geq \sum_{G \in \tilde{\mathcal{H}}} L_{G}$.

We now describe the effective constraints for any set $G=\bigcup_{G^{\prime} \in \mathcal{H}^{\prime}} G^{\prime}$ for some $\mathcal{H}^{\prime} \subset \tilde{\mathcal{H}}^{26}$ Let us denote such $\mathcal{H}^{\prime}$ as $\mathcal{H}_{G}$. The effective lower bound for $G=\bigcup_{G^{\prime} \in \mathcal{H}_{G}} G^{\prime}$ is $L(G)=\phi\left(\mathcal{H}_{G}\right):=$

\footnotetext{
${ }^{26}$ See the proof of Proposition 8 for the derivation of $C(G)$ and $L(G)$ for arbitrary $G$.
} 
$\max \left\{\sum_{G^{\prime} \in \mathcal{H}_{G}} L_{G^{\prime}}, L_{I}-\sum_{G^{\prime} \in \tilde{\mathcal{H}} \backslash \mathcal{H}_{G}} C_{G^{\prime}}\right\}$. Obviously, $L(G)$ cannot be lower than the direct lower bound $\sum_{G^{\prime} \in \mathcal{H}_{G}} L_{G^{\prime}}$, but it can be strictly larger. If the maximal capacity allowed for groups in $\tilde{\mathcal{H}} \backslash \mathcal{H}_{G}$ is smaller than $L_{I}$, the indirect lower bound $L_{I}-\sum_{G^{\prime} \in \tilde{\mathcal{H}} \backslash \mathcal{H}_{G}} C_{G^{\prime}}$ may exceed the direct lower bound. Similarly, the effective upper bound for $G$ is clearly no greater than $\sum_{G^{\prime} \in \mathcal{H}_{G}} C_{G}^{\prime}$, but it can be strictly less. The agents in $G$ cannot get more than what is left after accommodating the lower bound for agents in $I \backslash G=\bigcup_{G^{\prime} \in\left(\tilde{\mathcal{H}} \backslash \mathcal{H}_{G}\right)} G^{\prime}$. Hence, the effective upper bound is given by $C(G)=\psi\left(\mathcal{H}_{G}\right):=\min \left\{\sum_{G^{\prime} \in \mathcal{H}_{G}} C_{G^{\prime}}, C_{I}-\sum_{G^{\prime} \in \tilde{\mathcal{H}} \backslash \mathcal{H}_{G}} L_{G^{\prime}}\right\}$.

We will show how our characterization of reduced-form auctions simplifies in this environment. Specifically, we shall characterize the interim allocation rules $Q=\left(Q_{i}\right)_{i \in I}$, where $Q_{i}: \Theta_{i} \rightarrow[0, n]$ that are reduced forms of ex-post allocation rules that respect $\left(C_{G}, L_{G}\right)_{G \in \mathcal{H}}$. By a standard argument, incentive compatibility implies that we can without loss restrict attention to a monotonic $Q$ where $Q_{i}$ is nondecreasing. For each $\theta \in \Theta$ and $G \in \mathcal{H}$, let $\mathcal{F}_{G}(\theta):=\prod_{i \in G} F_{i}\left(\theta_{i}\right)$ denote the probability that every agent $i \in G$ has a type less than or equal to $\theta_{i}$. Further, when the environment is group-symmetric in the sense that $F_{i}=F_{j}=: F_{G}, \underline{\theta}_{i}=\underline{\theta}_{j}=: \underline{\theta}_{G}, \bar{\theta}_{i}=\bar{\theta}_{j}=: \bar{\theta}_{G}$, for all $i, j \in G$ for each $G \in \tilde{\mathcal{H}}$, then it is useful (and often without loss) to consider a group-symmetric $Q$, where $Q_{i}=Q_{j}=: Q_{G}$ for all $i, j \in G$ for each $G \in \tilde{\mathcal{H}}$.

Given independence of types, we invoke the upper contour set characterization (Theorem 6 and Corollary 3). Our characterization is then simplified as follows:

Theorem 8. (i) A monotonic interim allocation $Q$ is implementable if and only if, for each $\theta=\left(\theta_{i}\right)_{i \in I} \in \Theta$,

$$
\sum_{i \in I} \int_{\theta_{i}}^{\bar{\theta}_{i}} Q_{i}\left(s_{i}\right) d F_{i}\left(s_{i}\right) \leq \sum_{\mathcal{H}^{\prime} \subset \mathcal{H}}\left(\psi\left(\mathcal{H}^{\prime}\right) \cdot \prod_{G \in \mathcal{H}^{\prime}}\left(1-\mathcal{F}_{G}(\theta)\right) \cdot \prod_{G \in \tilde{\mathcal{H}} \backslash \mathcal{H}^{\prime}} \mathcal{F}_{G}(\theta)\right),
$$

where $\psi\left(\mathcal{H}^{\prime}\right):=\min \left\{\sum_{G \in \mathcal{H}^{\prime}} C_{G}, C_{I}-\sum_{G \in \tilde{\mathcal{H}} \backslash \mathcal{H}^{\prime}} L_{G}\right\}$ and,

$$
\sum_{i \in I} \int_{\underline{\theta}_{i}}^{\theta_{i}} Q_{i}\left(s_{i}\right) d F_{i}\left(s_{i}\right) \geq \sum_{\mathcal{H}^{\prime} \subset \tilde{\mathcal{H}}}\left(\phi\left(\mathcal{H}^{\prime}\right) \cdot \prod_{G \in \mathcal{H}^{\prime}} \mathcal{F}_{G}(\theta) \cdot \prod_{G \in \tilde{\mathcal{H}} \backslash \mathcal{H}^{\prime}}\left(1-\mathcal{F}_{G}(\theta)\right)\right),
$$

where $\phi\left(\mathcal{H}^{\prime}\right):=\max \left\{\sum_{G \in \mathcal{H}^{\prime}} L_{G}, L_{I}-\sum_{G \in \tilde{\mathcal{H}} \backslash \mathcal{H}^{\prime}} C_{G}\right\}$.

(ii) In a group-symmetric environment, a group-symmetric $Q=\left(Q_{G}\right)_{G \in \tilde{\mathcal{H}}}$ is a reduced form if and only if for each $\left(\theta_{G}\right)_{G \in \tilde{\mathcal{H}}} \in \times_{G \in \tilde{\mathcal{H}}}\left[\underline{\theta}_{G}, \bar{\theta}_{G}\right]$,

$$
\sum_{G \in \tilde{\mathcal{H}}}|G| \int_{\theta_{G}}^{\bar{\theta}_{G}} Q_{G}(s) d F_{G}(s) \leq \sum_{\mathcal{H}^{\prime} \subset \tilde{\mathcal{H}}}\left(\psi\left(\mathcal{H}^{\prime}\right) \cdot \prod_{G \in \mathcal{H}^{\prime}}\left(1-\left(F_{G}\left(\theta_{G}\right)\right)^{|G|}\right) \cdot \prod_{G \in \tilde{\mathcal{H}} \backslash \mathcal{H}^{\prime}}\left(F_{G}\left(\theta_{G}\right)\right)^{|G|}\right),
$$


and

$$
\sum_{G \in \tilde{\mathcal{H}}}|G| \int_{\underline{\theta}_{G}}^{\theta_{G}} Q_{G}(s) d F_{G}(s) \geq \sum_{\mathcal{H}^{\prime} \subset \tilde{\mathcal{H}}}\left(\phi\left(\mathcal{H}^{\prime}\right) \cdot \prod_{G \in \mathcal{H}^{\prime}}\left(F_{G}\left(\theta_{G}\right)\right)^{|G|} \cdot \prod_{G \in \tilde{\mathcal{H}} \backslash \mathcal{H}^{\prime}}\left(1-\left(F_{G}\left(\theta_{G}\right)\right)^{|G|}\right)\right) .
$$

The proof of this result can be found in Section A.3 in the Supplementary Material. The conditions for feasibility are explained as follows. The condition $\left(B U^{\prime}\right)$ requires that the total quantity allocated to the agents with types in upper contour sets $T=\bigsqcup_{i \in I}\left[\theta_{i}, \bar{\theta}_{i}\right]$, for each $\theta=\left(\theta_{i}\right)$, should not exceed the expected upper bounds for those agents who have types in $T$-more precisely, the upper bound for each possible family $\mathcal{H}^{\prime}$ of groups of agents multiplied by the probability that for each group in the family at least one agent has a type from $T$ and for all other groups, no agent has a type in $T$. Meanwhile, $\left(B L^{\prime}\right)$ requires that the total quantity allocated to the agents with types in lower contour sets $T=\bigsqcup_{i \in I}\left[\underline{\theta}_{i}, \theta_{i}\right]$, for each $\theta=\left(\theta_{i}\right)$, cannot be less than the expected lower bounds for those agents who have types in $T$.

We now derive characterization of reduced-form auctions for a variety of situations.

4.1. Individual Constraints. One simple case of interest is individual capacity constraints. An individual constraint may arise from a firm's preferences or technologies. For instance, a firm targeting a regional market with limited demand is unlikely to demand more than a certain number of licenses. Similarly, the individual constraint may come from a firm's limited technological capacity to utilize licenses. To be specific, suppose each firm $i \in I$ demands or can obtain at most $C_{i}$ units of the good, and these are the only type of constraints present. (In particular, lower bounds equal zero.) This case is a special case of a partitional constraint structure where each $G \in \tilde{\mathcal{H}}$ is a singleton set. Theorem 8 yields the following results as corollary.

Corollary 4. (i) A monotonic interim allocation $Q$ is a reduced form of an allocation satisfying individual constraints $\left(C_{i}\right)_{i \in G}$, if and only if, for each $\theta=\left(\theta_{i}\right)_{i \in I} \in \Theta$,

$$
\sum_{i \in I} \int_{\theta_{i}}^{\bar{\theta}_{i}} Q_{i}\left(s_{i}\right) d F_{i}\left(s_{i}\right) \leq \sum_{I^{\prime} \subset I}\left(\min \left\{\sum_{i \in I^{\prime}} C_{i}, n\right\} \cdot \prod_{i \in I^{\prime}}\left(1-F_{i}\left(\theta_{i}\right)\right) \cdot \prod_{i \notin I^{\prime}} F_{i}\left(\theta_{i}\right)\right) .
$$

(ii) If the agents are symmetric with $F_{i}=: F$ and $C_{i}=: m$, then a symmetric interim allocation rule $(Q, . ., Q)$ is a reduced form satisfying an individual constraint of $m$, if and only if, for each $\theta \in[\underline{\theta}, \bar{\theta}]$,

$$
|I| \int_{\theta}^{\bar{\theta}} Q\left(s_{i}\right) d F_{i}\left(s_{i}\right) \leq \sum_{k=0}^{|I|} \min \{k \cdot m, n\}\left(\begin{array}{c}
|I| \\
k
\end{array}\right)(1-F(\theta))^{k} F(\theta)^{|I|-k} .
$$


The latter condition is particularly intuitive. It says the total quantity accruing to the agents with types above $\theta$ must not exceed the probability that exactly $k$ agents have types above $\theta$ multiplied by the upper bound $\min \{k \cdot m, n\}$ these agents will face.

4.2. Group-Specific Quotas. An auction designer often wishes to limit the number of units allocated to a group of agents. An important motivation for doing so may come from an anti-trust consideration. If important rights such as licenses are concentrated to dominant firms, then it may be in the social interest to keep them from accumulating more. A group specific quota may also be used to protect domestic firms from competition by foreign firms, or to protect minority participants in auctions.

These scenarios are modeled as a special case of a partitional structure. The bidders $I$ are partitioned into two groups, $A$ and $B$, such that bidders in $A$ (e.g., incumbent, foreign, or non-minority firms) are subject to a cap $m<n$, while no such restriction applies to group $B$ of bidders. Again the reduced form characterization in these cases follows easily from Theorem 8.

Corollary 5. (i) A monotonic interim allocation $Q$ is a reduced form of an allocation satisfying group-specific quotas $(m, n)$, if and only if, for each $\theta=\left(\theta_{i}\right)_{i \in I} \in \Theta$,

$$
\sum_{i \in I} \int_{\theta_{i}}^{\bar{\theta}_{i}} Q_{i}\left(s_{i}\right) d F_{i}\left(s_{i}\right) \leq m\left(1-\prod_{i \in A} F_{i}\left(\theta_{i}\right)\right) \prod_{j \in B} F_{j}\left(\theta_{j}\right)+n\left(1-\prod_{j \in B} F_{j}\left(\theta_{j}\right)\right) .
$$

(ii) If the agents in each group are symmetric with $F_{i}=: F_{A}$ for $i \in A$ and $F_{j}=: F_{B}$ for $j \in B$, then a symmetric interim allocation rule $\left(Q_{A}, . ., Q_{A}, Q_{B}, \ldots, Q_{B}\right)$ is a reduced form satisfying the quotas $(m, n)$, if and only if, for each $\theta_{A} \in\left[\underline{\theta}_{A}, \bar{\theta}_{A}\right]$ and $\theta_{B} \in\left[\underline{\theta}_{B}, \bar{\theta}_{B}\right]$,

$|A| \int_{\theta_{A}}^{\bar{\theta}} Q_{A}(s) d F_{A}(s)+|B| \int_{\theta_{B}}^{\bar{\theta}} Q_{B}(s) d F_{B}(s) \leq m\left(1-F_{A}\left(\theta_{A}\right)^{|A|}\right) F_{B}\left(\theta_{B}\right)^{|B|}+n\left(1-F_{B}\left(\theta_{B}\right)^{|B|}\right)$.

Intuitively, the conditions state that, for any profile of upper-tail type intervals, the expected number units allocated to bidders in these type sets cannot exceed $m$ multiplied by the probability that no bidders in $B$ have types in these sets and some bidders in $A$ have types in these sets, plus $n$ multiplied by the probability that bidders in $B$ have types in these sets.

4.3. Partnership Dissolution, Spectrum Reallocation, and Set-Asides. The applications so far do not involve lower bound constraints. Lower bound constraints are relevant in a number of settings. For instance, in a partnership dissolution problem, an object in question - a physical asset or a corporation as a going concern - is allocated among partners, so unlike in the standard auction problem the object is always allocated to some partner (Cramton et al., 1987). A similar feature exists in designing a mechanism that reallocates 
licenses from existing (inefficient) users to new (more productive) users, as mentioned in Section 2.2. This problem introduces a lower bound constraint on the number of licenses to be allocated to the whole set of players. A similar constraint is present in a government auction in which the government is committed to allocate a predetermined number of licenses.

Formally, the problem has a partitional constraint structure with the coarsest partition: $\mathcal{H}=\tilde{\mathcal{H}}=\{I\}$, and $C_{I}=L_{I}=n$. That is, all $n$ units must be allocated to some bidders. The following characterization then follows from Theorem 8.

Corollary 6. A monotonic interim allocation $Q$ is a reduced form of an allocation satisfying $\left(C_{I}, L_{I}\right)=(n, n)$, if and only if, for each $\theta=\left(\theta_{i}\right)_{i \in I} \in \Theta$,

$$
\sum_{i \in I} \int_{\theta_{i}}^{\bar{\theta}_{i}} Q_{i}\left(s_{i}\right) d F_{i}\left(s_{i}\right) \leq n\left(1-\prod_{j \in I} F_{j}\left(\theta_{j}\right)\right)
$$

and

$$
\sum_{i \in I} \int_{\underline{\theta}_{i}}^{\bar{\theta}_{i}} Q_{i}\left(s_{i}\right) d F_{i}\left(s_{i}\right)=n
$$

The first part is the condition familiar from Border (1991). The second part states an obvious necessary condition that the entire units must be allocated to all agents. Remarkably, this latter condition, together with the first part, is also sufficient for the lower bound constraints. As proven in Theorem 8, the lower bound condition requires that for each $\theta=\left(\theta_{i}\right)_{i \in I} \in \Theta$,

$$
\sum_{i \in I} \int_{\underline{\theta}_{i}}^{\theta_{i}} Q_{i}\left(s_{i}\right) d F_{i}\left(s_{i}\right) \geq n \prod_{j \in I} F_{j}\left(\theta_{j}\right)
$$

Clearly, this condition is implied by the pair of conditions required by Corollary 6 .

A lower bound constraint is also relevant in some government auctions where some units of licenses are set aside for some designated group of buyers (see Pai and Vohra, 2012; Athey et al., 2013). Protecting/promoting minority interests can take the form of capping the maximum number of units allocated to the bidders "outside" the designated group. This can be handled simply by upper bound constraints, as seen above. A more "active" form of set-aside sale would involve a lower bound on the units allocated to the designated group. Recall the scenario discussed in Section 4.2. Suppose instead of limiting the amount of the good allocated for group $\mathrm{A}$, the target group $B$ is now protected by the minimum amount $k=n-m$ of the good. In that case, the reduced form is characterized as follows.

Corollary 7. A monotonic interim allocation $Q$ is a reduced form of an allocation satisfying $L_{B}=k$, if and only if, for each $\theta=\left(\theta_{i}\right)_{i \in I} \in \Theta$,

$$
\sum_{i \in I} \int_{\theta_{i}}^{\bar{\theta}_{i}} Q_{i}\left(s_{i}\right) d F_{i}\left(s_{i}\right) \leq(n-k)\left(1-\prod_{i \in A} F_{i}\left(\theta_{i}\right)\right) \prod_{j \in B} F_{j}\left(\theta_{j}\right)+n\left(1-\prod_{j \in B} F_{j}\left(\theta_{j}\right)\right) .
$$


and

$$
\sum_{i \in B} \int_{\underline{\theta}_{i}}^{\theta_{i}} Q_{i}\left(s_{i}\right) d F_{i}\left(s_{i}\right) \geq k \prod_{j \in B} F_{j}\left(\theta_{j}\right) .
$$

\section{Appendix: Omitted Proofs}

Proof of Lemma 1. Assume $\mathcal{P}$ is nonempty. To prove paramodularity of $(C, L)$, we first prove that its restriction $\left.(C, L)\right|_{\mathcal{H}}$ to sets in $\mathcal{H}$ is paramodular. To begin, we prove that $\left.C\right|_{\mathcal{H}}$ is submodular. To this end, fix any $G, G^{\prime} \in \mathcal{H}$. Since $\mathcal{H}$ is a hierarchy, $G \subset G^{\prime}$ or $G^{\prime} \subset G$ or $G \cap G^{\prime}=\emptyset$. If $G \subset G^{\prime}$ or $G^{\prime} \subset G$, then the submodularity condition is vacuous, so assume $G \cap G^{\prime}=\emptyset$. Let $x^{*}=\left(x_{1}^{*}, \ldots, x_{|I|}^{*}\right)$ be a maximizer that solves $\max \left\{\sum_{i \in G \cup G^{\prime}} x_{i} \mid x \in \mathcal{P}\right\}$. Then, since $x^{*} \in \mathcal{P}$, we must have

$$
C(G)=\max \left\{\sum_{i \in G} x_{i} \mid x \in \mathcal{P}\right\} \geq \sum_{i \in G} x_{i}^{*} \text { and } C\left(G^{\prime}\right)=\max \left\{\sum_{i \in G^{\prime}} x_{i} \mid x \in \mathcal{P}\right\} \geq \sum_{i \in G^{\prime}} x_{i}^{*} .
$$

Hence, since $G \cap G^{\prime}=\emptyset$,

$$
C(G)+C\left(G^{\prime}\right) \geq \sum_{i \in G} x_{i}^{*}+\sum_{i \in G^{\prime}} x_{i}^{*}=\sum_{i \in G \cup G^{\prime}} x_{i}^{*}=C\left(G \cup G^{\prime}\right)+C(\emptyset),
$$

proving the submodularity of $\left.C\right|_{\mathcal{H}}$. The argument for the supermodularity of $\left.L\right|_{\mathcal{H}}$ is completely symmetric. To prove that $\left.(C, L)\right|_{\mathcal{H}}$ is compliant, suppose not. Then, there exist $G^{\prime}, G$ such that

$$
C\left(G^{\prime}\right)-L(G)<C\left(G^{\prime} \backslash G\right)-L\left(G \backslash G^{\prime}\right) .
$$

This cannot happen if $G \cap G^{\prime}=\emptyset$. So suppose first $G^{\prime} \supset G$, then (11) reduces to

$$
C\left(G^{\prime}\right)-L(G)<C\left(G^{\prime} \backslash G\right) .
$$

Let $x^{*} \in \mathcal{P}$ be a maximizer that solves $\max \left\{\sum_{i \in G^{\prime} \backslash G} x_{i} \mid x \in \mathcal{P}\right\}=C\left(G^{\prime} \backslash G\right)$. Since $x^{*} \in \mathcal{P}$,

$$
C\left(G^{\prime}\right)-L(G) \geq \sum_{i \in G^{\prime} \backslash G} x_{i}^{*}=C\left(G^{\prime} \backslash G\right),
$$

which contradicts (12). A symmetric argument yields a contradiction if $G^{\prime} \subset G$. Combining the observations, we conclude that $\left.(C, L)\right|_{\mathcal{H}}$ is paramodular.

The paramodularity of $(C, L)$ as well as the claim that the feasible set defined by $(C, L)$ coincides with $\mathcal{P}$ then follows from Theorem 49.13 of Schrijver (2000).

Proof of Theorem 1. ("If" part). Suppose the network $(N, E, k, d)$ admits a feasible circulation flow $f$. Then, define $q_{i}(\theta)=\frac{f\left(\theta, \theta_{i}\right)}{p(\theta)}$ for each $\theta \in \Theta$ and $i \in I$. Note first that $q$ respects $(C, L)$ since, for any $G \subset I$,

$$
\sum_{i \in G} q_{i}(\theta)=\sum_{i \in G} \frac{f\left(\theta, \theta_{i}\right)}{p(\theta)} \in\left[\frac{d\left(\theta,\left\{\theta_{i}\right\}_{i \in G}\right)}{p(\theta)}, \frac{k\left(\theta,\left\{\theta_{i}\right\}_{i \in G}\right)}{p(\theta)}\right]=[L(G), C(G)]
$$


where the inclusion relationship holds since $f$ satisfies the lower/upper bound constraints while the last equality holds since $I\left(\theta,\left\{\theta_{i}\right\}_{i \in G}\right)=G$. Also, the flow conservation law implies that for each $\theta_{i} \in D$,

$$
p_{i}\left(\theta_{i}\right) Q_{i}\left(\theta_{i}\right)=f\left(\theta_{i}, N \backslash\left\{\theta_{i}\right\}\right)=f\left(N \backslash\left\{\theta_{i}\right\}, \theta_{i}\right)=\sum_{\tilde{\theta} \in \Theta: \tilde{\theta}_{i}=\theta_{i}} f\left(\tilde{\theta}, \theta_{i}\right)=\sum_{\tilde{\theta}_{-i} \in \Theta_{-i}} p\left(\theta_{i}, \tilde{\theta}_{-i}\right) q_{i}\left(\theta_{i}, \tilde{\theta}_{-i}\right),
$$

meaning that $Q$ is the reduced form of $q$.

("Only if" part) Suppose that the interim allocation rule $Q$ is the reduced form of an allocation rule $q$ that respects $(C, L)$. We can then construct a feasible circulation flow for the above network as follows: for each $\tilde{\theta} \in \Theta$ and $\theta_{i} \in D$ with $\tilde{\theta}_{i}=\theta_{i}, f\left(\tilde{\theta}, \theta_{i}\right)=p(\tilde{\theta}) q_{i}(\tilde{\theta})$, for each $\theta_{i} \in D, f\left(\theta_{i}, t\right)=p_{i}\left(\theta_{i}\right) Q_{i}\left(\theta_{i}\right)$, and for each $\theta \in \Theta, f(t, \theta)=\sum_{i \in I} p(\theta) q_{i}(\theta)$. We prove that this flow satisfies the flow conservation law and lower/upper bound constraints.

First, for each supply node $\theta \in \Theta$, we have $f(\theta, N \backslash\{\theta\})=f(N \backslash\{\theta\}, \theta)=\sum_{i \in I} p(\theta) q_{i}(\theta)$. Also, for any $N^{\prime} \subset N \backslash\{\theta\}$,

$$
f\left(\theta, N^{\prime}\right)=\sum_{i \in I\left(\theta, N^{\prime} \cap D\right)} p(\theta) q_{i}(\theta) \in\left[p(\theta) L\left(I\left(\theta, N^{\prime} \cap D\right)\right), p(\theta) C\left(I\left(\theta, N^{\prime} \cap D\right)\right)\right]=\left[d\left(\theta, N^{\prime}\right), k\left(\theta, N^{\prime}\right)\right]
$$

since $q$ respects $(C, L)$, which means that $f(\theta, \cdot)$ satisfies the lower/upper bound constraints.

Second, for each demand node $\theta_{i}$, we have $f\left(\theta_{i}, N \backslash\left\{\theta_{i}\right\}\right)=p_{i}\left(\theta_{i}\right) Q_{i}\left(\theta_{i}\right)$ and $f\left(N \backslash\left\{\theta_{i}\right\}, \theta_{i}\right)=$ $\sum_{\tilde{\theta} \in \Theta: \tilde{\theta}_{i}=\theta_{i}} f\left(\tilde{\theta}, \theta_{i}\right)=\sum_{\tilde{\theta}_{-i} \in \Theta_{-i}} p\left(\theta_{i}, \tilde{\theta}_{-i}\right) q_{i}\left(\theta_{i}, \tilde{\theta}_{-i}\right)$. Then, the flow conservation law is satisfied since $Q$ is the reduced form of $q$. Also, the lower/upper bound constraints for the flows $f\left(\theta_{i}, \cdot\right)$ are satisfied since, for any $N^{\prime} \subset N \backslash\left\{\theta_{i}\right\}$ with $t \in N^{\prime}, f\left(\theta_{i}, N^{\prime}\right)=p_{i}\left(\theta_{i}\right) Q_{i}\left(\theta_{i}\right)=k\left(\theta_{i}, N^{\prime}\right)=$ $d\left(\theta_{i}, N^{\prime}\right)$.

Lastly, for the circulation node $n=t$, we have

$$
f(t, N \backslash\{t\})=\sum_{\theta \in \Theta} \sum_{i \in I} p(\theta) q_{i}(\theta)=\sum_{\theta_{i} \in D} p_{i}\left(\theta_{i}\right) Q_{i}\left(\theta_{i}\right)=f(t, N \backslash\{t\}),
$$

where the second equality again follows from $Q$ being the reduced form of $q$. The lower/upper bound constraints are trivially satisfied for the flows $f(t, \cdot)$ since $K$ is sufficiently large. Hence, $f$ is a feasible circulation flow.

Proof of Theorem 3. NECESSITY: Suppose that the interim allocation rule $Q$ is the reduced form of an allocation rule $q$ that respects $(C, L)$. Then, by Theorem 1 , the network $(N, E, k, d)$ admits a feasible circulation flow $f$. Hence, $f$ must satisfy (6) for any $M \subset N$, by Theorem 2. Consider any $T \subset D$, let $M=T$. The RHS of (6) becomes

$$
\sum_{n \in M} k(n, N \backslash M)=\sum_{\theta_{i} \in T} k\left(\theta_{i}, t\right)=\sum_{\theta_{i} \in T} p_{i}\left(\theta_{i}\right) Q_{i}\left(\theta_{i}\right)=\sum_{i \in I} \sum_{\theta_{i} \in T_{i}} p_{i}\left(\theta_{i}\right) Q_{i}\left(\theta_{i}\right)
$$


and the LHS of (6) becomes

$$
\sum_{n \in N \backslash M} d(n, M)=\sum_{n \in N \backslash T} d(n, T)=\sum_{\theta \in Y(T)} d(\theta, T)=\sum_{\theta \in Y(T)} p(\theta) L(I(\theta, T)),
$$

which gives us the first inequality in $\left(B^{\prime}\right)$. Now let $M=N \backslash T$. The RHS of (6) becomes

$$
\sum_{n \in M} k(n, N \backslash M)=\sum_{n \in N \backslash T} k(n, T)=\sum_{\theta \in Y(T)} k(\theta, T)=\sum_{\theta \in Y(T)} p(\theta) C(I(\theta, T))
$$

and the LHS of (6) becomes

$$
\sum_{n \in N \backslash M} d(n, M)=\sum_{\theta_{i} \in T} d\left(\theta_{i}, N \backslash T\right)=\sum_{\theta_{i} \in T} d\left(\theta_{i}, t\right)=\sum_{i \in I} \sum_{\theta_{i} \in T_{i}} p_{i}\left(\theta_{i}\right) Q_{i}\left(\theta_{i}\right),
$$

which gives us the second inequality in $\left(B^{\prime}\right)$. This completes the proof of necessity of $\left(B^{\prime}\right)$.

Sufficiency: We now show that $\left(B^{\prime}\right)$ implies (6). We consider two cases depending on whether $t \in M$ or not.

Suppose first that $t \notin M$. In this case,

$$
\begin{aligned}
\sum_{n \in N \backslash M} d(n, M) & =d(t, M \cap \Theta)+\sum_{n \in N \backslash(M \cup\{t\})} d(n, M) \\
& =\sum_{n \in \Theta \backslash M} d(n, M \cap D) \leq \sum_{\theta \in Y(M \cap D)} d(n, M \cap D) \\
& =\sum_{\theta \in Y(M \cap D)} p(\theta) L(I(\theta, M \cap D)) \leq \sum_{\theta_{i} \in M \cap D} p_{i}\left(\theta_{i}\right) Q_{i}\left(\theta_{i}\right) \\
& \leq \sum_{\theta \in M \cap \Theta} C(I(\theta, D \backslash M)) p(\theta)+\sum_{\theta_{i} \in D \cap M} k\left(\theta_{i}, t\right)=\sum_{n \in M} k(n, N \backslash M) .
\end{aligned}
$$

Suppose next that $t \in M$. Then, if $\Theta \nsubseteq M$, then we have $\sum_{n \in M} k(n, N \backslash M) \geq k(t, \Theta \backslash$ $M)=K>\sum_{n \in N \backslash M} d(n, M)$ for $K$ sufficiently large. Otherwise, if $\Theta \subset M$,

$$
\begin{aligned}
\sum_{n \in M} k(n, N \backslash M) & =\sum_{\theta \in \Theta} k(\theta, D \backslash M)=\sum_{\theta \in Y(D \backslash M)} p(\theta) C(I(\theta, D \backslash M)) \\
& \geq \sum_{\theta_{i} \in D \backslash M} p_{i}\left(\theta_{i}\right) Q_{i}\left(\theta_{i}\right)=\sum_{\theta_{i} \in D \backslash M} d\left(\theta_{i}, t\right)=\sum_{n \in N \backslash M} d(n, M)
\end{aligned}
$$

To sum up, if $\left(B^{\prime}\right)$ holds, then (6) also holds, so there exists a feasible circulation flow $f$. The conclusion then follows by Theorem 1.

Proof of Theorem 6. Necessity is obvious. To establish sufficiency, we only consider $(B U)$. The argument for $(B L)$ is completely symmetric and is omitted. To begin, fix any agent $i$ and arbitrary type sets $T_{j} \subset \Theta_{j} . j \neq i$. Then, for any $T_{i} \subset \Theta_{i},\left(B^{\prime}\right)$ becomes

$$
\sum_{j \in I} \sum_{\theta_{j} \in T_{j}} q_{j}\left(\theta_{j}\right) p_{j}(\theta) \leq \sum_{\theta \in Y(T)} C(I(\theta, T)) p(\theta)
$$




$$
\begin{aligned}
& =\underbrace{\sum_{\theta \in \bigcup_{j \neq i}\left(T_{j} \times \Theta_{-j}\right)} C(I(\theta, T)) p(\theta)}_{=\alpha_{i}(T)}+\sum_{\theta \in\left(T_{i} \times \Theta_{-i}\right) \backslash \bigcup_{j \neq i}\left(T_{j} \times \Theta_{-j}\right)} C(I(\theta, T)) p(\theta) \\
& =\alpha_{i}(T)+\sum_{\theta_{i} \in T_{i}} \beta_{i}\left(T_{-i}\right) C(\{i\}) p_{i}\left(\theta_{i}\right),
\end{aligned}
$$

where $\beta_{i}\left(T_{-i}\right)=\prod_{j \neq i}\left(1-p_{j}\left(T_{j}\right)\right)$. The second equality holds because in the second sum in the second line, we have $\theta_{i} \in T_{i}$ and $\theta_{j} \notin T_{j}$ for all $j \neq i$. But this implies that $I(\theta, T)=\{i\}$, independent of $\theta_{-i}$. Using independence of the type distributions, we get

$$
\sum_{\theta \in\left(T_{i} \times \Theta_{-i}\right) \backslash \bigcup_{j \neq i}\left(T_{j} \times \Theta_{-j}\right)} C(\{i\}) p(\theta)=\sum_{\theta_{i} \in T_{i}} \beta_{i}\left(T_{-i}\right) C(\{i\}) p_{i}\left(\theta_{i}\right) .
$$

We now rewrite $\left(B^{\prime}\right)$ as

$$
\Upsilon\left(T_{i}, T_{-i}\right):=\sum_{\theta_{i} \in T_{i}}\left(q_{i}\left(\theta_{i}\right)-\beta_{i}\left(T_{-i}\right) C(\{i\})\right) p_{i}\left(\theta_{i}\right)-\alpha_{i}(T) \leq-\sum_{j \neq i} \sum_{\theta_{j} \in T_{j}} q_{j}\left(\theta_{j}\right) p_{j}\left(\theta_{j}\right) .
$$

For the proof, it will then suffice to show that for given $T_{-i}, \Upsilon\left(T_{i}, T_{-i}\right)$ is maximized by a set $T_{i}$ that is an upper contour set of $q_{i} \cdot{ }^{27}$

To begin, we establish the following property of $\alpha_{i}(\cdot)$.

Claim 1. For any set $T_{i}$ and any $\tilde{\theta}_{i} \in T_{i}$, let $\tilde{T}_{i}=T_{i} \backslash\left\{\tilde{\theta}_{i}\right\}$ and $\tilde{T}=\left(\tilde{T}_{i}, T_{-i}\right)$. Then, there is some $\gamma_{i}\left(T_{-i}\right) \geq 0$ such that

$$
\alpha_{i}(T)-\alpha_{i}(\tilde{T})=\gamma_{i}\left(T_{-i}\right) p_{i}\left(\tilde{\theta}_{i}\right)
$$

Proof. Using the definition of $\alpha_{i}(\cdot)$, we have

$$
\alpha_{i}(T)-\alpha_{i}(\tilde{T})=\sum_{\theta \in \bigcup_{j \neq i}\left(T_{j} \times \Theta_{-j}\right)}[C(I(\theta, T))-C(I(\theta, \tilde{T}))] p(\theta) .
$$

If $\theta$ is such that $\theta_{i} \neq \tilde{\theta}_{i}, I(\theta, T)=I(\theta, \tilde{T})$. Hence

$$
\begin{aligned}
\alpha_{i}(T)-\alpha_{i}(\tilde{T}) & =\sum_{\theta \in \bigcup_{j \neq i}\left(T_{j} \times\left\{\tilde{\theta}_{i}\right\} \times \Theta_{-i j}\right)}[C(I(\theta, T))-C(I(\theta, \tilde{T}))] p(\theta) \\
& =(\underbrace{\sum_{\theta_{-i} \in \bigcup_{j \neq i}\left(T_{j} \times \Theta_{-i j}\right)}\left[C\left(I\left(\left(\tilde{\theta}_{i}, \theta_{-i}\right), T\right)\right)-C\left(I\left(\left(\tilde{\theta}_{i}, \theta_{-i}\right), \tilde{T}\right)\right)\right] p_{-i}\left(\theta_{-i}\right)}_{=: \gamma_{i}\left(T_{-i}\right)}) p_{i}\left(\tilde{\theta}_{i}\right),
\end{aligned}
$$

We now argue that the expression in the large parentheses is independent of $T_{i}$ and $\tilde{\theta}_{i}$. For any choice of $T_{i}$ and $\tilde{\theta}_{i}, I\left(\left(\tilde{\theta}_{i}, \theta_{-i}\right), T\right)=\left\{j \neq i \mid \theta_{j} \in T_{j}\right\} \cup\{i\}$ because $\tilde{\theta}_{i} \in T_{i}$, and

$\overline{{ }^{27} \text { The original }}$ idea of this proof is from Theorem 4 in Gutmann et al. (1991). 
$I\left(\left(\tilde{\theta}_{i}, \theta_{-i}\right), \tilde{T}\right)=I\left(\left(\tilde{\theta}_{i}, \theta_{-i}\right), T\right) \backslash\{i\}$. This implies that $C\left(I\left(\left(\tilde{\theta}_{i}, \theta_{-i}\right), T\right)\right)$ and $C\left(I\left(\left(\tilde{\theta}_{i}, \theta_{-i}\right), \tilde{T}\right)\right)$ are independent of $T_{i}$ and $\tilde{\theta}_{i}$.

The claim implies that

$$
\Upsilon\left(T_{i}, T_{-i}\right)=\sum_{\theta_{i} \in T_{i}}\left[q_{i}\left(\theta_{i}\right)-\beta_{i}\left(T_{-i}\right) C(\{i\})-\gamma_{i}\left(T_{-i}\right)\right] p_{i}\left(\theta_{i}\right)-\alpha_{i}\left(\emptyset, T_{-i}\right) .
$$

Obviously, this expression is maximized by the upper contour set $T_{i}=\left\{\theta_{i} \in \Theta_{i} \mid q_{i}\left(\theta_{i}\right) \geq\right.$ $\left.\beta_{i}\left(T_{-i}\right) C(\{i\})+\gamma_{i}\left(T_{-i}\right)\right\}$.

Proof of Theorem 7. For the proof we introduce the following notation. For $T \subset D$, $T=\bigsqcup_{i \in I} T_{i}$, we rewrite the definitions in Remark 2 as $\tilde{\Psi}\left(T_{1}, \ldots T_{I}\right)=\Psi(T)$ and $\tilde{\Phi}\left(T_{1}, \ldots T_{I}\right)=$ $\Phi(T)$.

Claim 2. Due to the group-symmetry, both $\tilde{\Psi}\left(T_{1}, \ldots, T_{|I|}\right)$ and $\tilde{\Phi}\left(T_{1}, \ldots, T_{|I|}\right)$ are invariant to permutations of the sets $\left(T_{i}\right)_{i \in G_{\ell}}$.

Proof. Let us focus on $\tilde{\Psi}$. It suffices to consider a binary permutation $\pi: I \rightarrow I$ defined as $\pi(i)=j$ and $\pi(j)=i$ for some $i, j \in G_{\ell}$ with $\pi(k)=k$ for all $k \neq i, j$. Let $T^{\pi}:=\left(T_{\pi(k)}\right)_{k \in I}$ and $\theta^{\pi}:=\left(\theta_{\pi(k)}\right)_{k \in I}$. Note first that $p(\theta)=p\left(\theta^{\pi}\right)$. We next argue that $C(I(\theta, T))=C\left(I\left(\theta^{\pi}, T^{\pi}\right)\right)$. This is trivial in case either $i, j \in I(\theta, T)$ or $i, j \notin I(\theta, T)$, since then $I\left(\theta^{\pi}, T^{\pi}\right)=I(\theta, T)$. In case $i \in I(\theta, T)$ and $j \notin I(\theta, T)$, letting $I^{\prime}=I(\theta, T) \backslash\{i\}$, we have $C\left(I\left(\theta^{\pi}, T^{\pi}\right)\right)=C\left(I^{\prime} \cup\{j\}\right)=C\left(I^{\prime} \cup\{i\}\right)=C(I(\theta, T))$ by the group-symmetry. The argument is analogous in case $i \notin I(\theta, T)$ and $j \in I(\theta, T)$. Then,

$\tilde{\Psi}\left(T^{\pi}\right)=\sum_{\theta^{\prime} \in Y\left(T^{\pi}\right)} C\left(I\left(\theta^{\prime}, T^{\pi}\right)\right) p\left(\theta^{\prime}\right)=\sum_{\theta \in Y(T)} C\left(I\left(\theta^{\pi}, T^{\pi}\right)\right) p\left(\theta^{\pi}\right)=\sum_{\theta \in Y(T)} C(I(\theta, T)) p(\theta)=\tilde{\Psi}(T)$,

where the second equality follows from the fact that $\theta^{\prime} \in Y\left(T^{\pi}\right)$ if and only if there is some $\theta \in Y(T)$ such that $\theta^{\prime}=\theta^{\pi}$.

Note now that for $T, T^{\prime} \subset D$, we have $T \cup T^{\prime}=\bigsqcup_{i \in I}\left(T_{i} \cup T_{i}^{\prime}\right)$ and $T \cap T^{\prime}=\bigsqcup_{i \in I}\left(T_{i} \cap\right.$ $\left.T_{i}^{\prime}\right) .{ }^{28}$ Therefore, submodularity of $\Psi$ and supermodularity of $\Phi$ (see Theorem 4), imply, respectively, that for all $T, T^{\prime} \subset D$ :

$$
\tilde{\Psi}\left(T_{1}, \ldots, T_{|I|}\right)+\tilde{\Psi}\left(T_{1}^{\prime}, \ldots, T_{|I|}^{\prime}\right) \geq \tilde{\Psi}\left(T_{1} \cup T_{1}^{\prime}, \ldots, T_{|I|} \cup T_{|I|}^{\prime}\right)+\tilde{\Psi}\left(T_{1} \cap T_{1}^{\prime}, \ldots, T_{|I|} \cap T_{|I|}^{\prime}\right),
$$

and

$$
\tilde{\Phi}\left(T_{1}, \ldots, T_{|I|}\right)+\tilde{\Phi}\left(T_{1}^{\prime}, \ldots, T_{|I|}^{\prime}\right) \leq \tilde{\Phi}\left(T_{1} \cup T_{1}^{\prime}, \ldots, T_{|I|} \cup T_{|I|}^{\prime}\right)+\tilde{\Phi}\left(T_{1} \cap T_{1}^{\prime}, \ldots, T_{|I|} \cap T_{|I|}^{\prime}\right) .
$$

Now suppose that there is some $T \subset D$ for which the right inequality in $\left(B^{\prime}\right)$ is violated. Suppose that $T$ is minimal in the sense that for all proper subsets $T^{\prime} \subsetneq T$, the right inequality

\footnotetext{
${ }^{28}$ The two equalities here hold since $T$ and $T^{\prime}$ are the disjoint unions of $T_{i}^{\prime}$ 's and $T_{i}^{\prime}$ 's, respectively.
} 
in $\left(B^{\prime}\right)$ is fulfilled. We will show that if $T$ is not group-symmetric, then there is a groupsymmetric set $\hat{T}$ for which $\left(B^{\prime}\right)$ is also violated. Specifically, for each $\ell \in\{1, \ldots, L\}$, we define $\bar{T}_{\ell}:=\bigcup_{i \in G_{\ell}} T_{i}$ where $\bar{T}_{\ell}$ is the usual (not disjoint) union of the sets $T_{i}$, i.e., $\bar{T}_{\ell} \subset \hat{\Theta}_{\ell}$. Using this, we define the group-symmetric set $\hat{T}:=\bigsqcup_{i \in I} \hat{T}_{i}$ by setting $\hat{T}_{i}:=\bar{T}_{\ell}$ for all $\ell \in\{1, \ldots, L\}$ and all $i \in G_{\ell}$.

To show that the right inequality in $\left(B^{\prime}\right)$ is violated for $\hat{T}$, we show that starting from $T$ we can successively add types to the sets $T_{i}$ to obtain a sequence of sets $T=S^{1} \subsetneq S^{2} \subsetneq \ldots \subsetneq$ $S^{M}=\hat{T}$ such that the right inequality in $\left(B^{\prime}\right)$ remains violated for all $S^{m}, m=1, \ldots, M$. The sequence is constructed inductively:

Step 1: Set $S^{1}:=T$.

Step m: If $S^{m-1}=\hat{T}$, STOP. Otherwise there must be a group $\ell \in\{1, \ldots, L\}$ and $k, j \in G_{\ell}$ such that at least one of the sets $A:=T_{j} \backslash S_{k}^{m-1}$ and $B:=T_{k} \backslash S_{j}^{m-1}$ is non-empty. Define $S^{m}:=\left(S_{j}^{m-1} \cup B, S_{k}^{m-1} \cup A, S_{-j k}^{m-1}\right)$ and iterate to Step $m+1$.

Since $I$ is finite the construction stops after a finite number of steps. It remains to show

Claim 3. If the right inequality in $\left(B^{\prime}\right)$ is violated for $S^{m}$, then it is also violated for $S^{m+1}$.

Proof. By construction, least one of the sets $A, B$ is non-empty. By assumption, $\left(B^{\prime}\right)$ is violated for $T$,

$$
\sum_{i \in I} \sum_{\theta_{i} \in T_{i}} Q_{i}\left(\theta_{i}\right) p_{i}\left(\theta_{i}\right)>\tilde{\Psi}\left(T_{1}, \ldots, T_{|I|}\right)
$$

and as $T$ is chosen minimally, we have

$$
\sum_{i \in I} \sum_{\theta_{i} \in T_{i}} Q_{i}\left(\theta_{i}\right) p_{i}\left(\theta_{i}\right)-\sum_{\theta_{j} \in A} Q_{j}\left(\theta_{j}\right) p_{j}\left(\theta_{j}\right)-\sum_{\theta_{k} \in B} Q_{k}\left(\theta_{k}\right) p_{k}\left(\theta_{k}\right) \leq \tilde{\Psi}\left(T_{j} \backslash A, T_{k} \backslash B, T_{-j k}\right) .
$$

Hence

$$
\sum_{\theta_{j} \in A} Q_{j}\left(\theta_{j}\right) p_{j}\left(\theta_{j}\right)+\sum_{\theta_{k} \in B} Q_{k}\left(\theta_{k}\right) p_{k}\left(\theta_{k}\right)>\tilde{\Psi}\left(T_{j}, T_{k}, T_{-j k}\right)-\tilde{\Psi}\left(T_{j} \backslash A, T_{k} \backslash B, T_{-j k}\right) .
$$

For the right inequality in $\left(B^{\prime}\right)$ for $S^{m+1}=\left(S_{j}^{m} \cup B, S_{k}^{m} \cup A, S_{-j k}^{m}\right)$, we have

$$
\begin{aligned}
& \sum_{i \in I} \sum_{\theta_{i} \in S_{i}^{m}} Q_{i}\left(\theta_{i}\right) p_{i}\left(\theta_{i}\right)+\sum_{\theta_{j} \in B} Q_{j}\left(\theta_{j}\right) p_{j}\left(\theta_{j}\right)+\sum_{\theta_{k} \in A} Q_{k}\left(\theta_{k}\right) p_{k}\left(\theta_{k}\right) \\
= & \sum_{i \in I} \sum_{\theta_{i} \in S_{i}^{m}} Q_{i}\left(\theta_{i}\right) p_{i}\left(\theta_{i}\right)+\sum_{\theta_{j} \in A} Q_{j}\left(\theta_{j}\right) p_{j}\left(\theta_{j}\right)+\sum_{\theta_{k} \in B} Q_{k}\left(\theta_{k}\right) p_{k}\left(\theta_{k}\right) \\
> & \tilde{\Psi}\left(S^{m}\right)+\tilde{\Psi}\left(T_{j}, T_{k}, T_{-j k}\right)-\tilde{\Psi}\left(T_{j} \backslash A, T_{k} \backslash B, T_{-j k}\right) \\
= & \tilde{\Psi}\left(S^{m}\right)+\tilde{\Psi}\left(T_{k}, T_{j}, T_{-j k}\right)-\tilde{\Psi}\left(T_{k} \backslash B, T_{j} \backslash A, T_{-j k}\right) \\
\geq & \tilde{\Psi}\left(S^{m}\right)+\tilde{\Psi}\left(S_{j}^{m} \cup B, S_{k}^{m} \cup A, S_{-j k}^{m}\right)-\tilde{\Psi}\left(S_{j}^{m}, S_{k}^{m}, S_{-j k}^{m}\right) \\
= & \tilde{\Psi}\left(S_{j}^{m+1}, S_{k}^{m+1}, S_{-j k}^{m+1}\right)
\end{aligned}
$$


The first equality follows from group-symmetry since $k, j \in G_{\ell}$ for some $\ell$. The strict inequality follows from (22) and the assumption that the right-hand side of $\left(B^{\prime}\right)$ is violated for $S^{m}$. The second equality holds by symmetry. The weak inequality follows from (20) since $S_{j}^{m} \cup T_{k}=S_{j}^{m} \cup B, S_{j}^{m} \cap T_{k}=T_{k} \backslash B, S_{k}^{m} \cup T_{j}=S_{k}^{m} \cup A$, and $S_{k}^{m} \cap T_{j}=T_{j} \backslash A$.

Virtually the same argument can be applied to the left inequality of $\left(B^{\prime}\right)$ using (21).

Proof of Corollary 3. Let $\mathcal{T}_{U}:=\left\{T \subset D \mid \forall i: T_{i}\right.$ is an upper contour subset of $\left.Q_{i}\right\}$ and $\mathcal{T}_{L}:=\left\{T \subset D \mid \forall i: T_{i}\right.$ is a lower contour subset of $\left.Q_{i}\right\}$. Then, from Theorem 6, we know that $\left(B^{\prime}\right)$ holds for all $T$ if and only if $(B U)$ and $(B L)$ hold for all $T \in \mathcal{T}_{U}$ and all $T^{\prime} \in \mathcal{T}_{L}$, respectively. As in the proof of Theorem 7 , if $(B U)$ is violated for a minimal set $T \in \mathcal{T}_{U}$, then it is also violated for the group-symmetric set $\hat{T}{ }^{29}$ Since each $\hat{T}_{i}$ is the union of upper contour sets, $(B U)$ is violated for a group-symmetric set $\hat{T} \in \mathcal{T}_{U}$. A similar argument applies to $(B L)$.

\section{REFERENCES}

Alaei, S., H. Fu, N. Haghpanah, J. Hartline, and A. Malekian (2012): "Bayesian Optimal Auctions via Multi- to Single-agent Reduction," Unpublished manuscript.

Armstrong, M. (2000): "Optimal Multi-Object Auctions," Review of Economic Studies, $67,455-481$.

Asker, J. And E. Cantillon (2010): "Procurement when Price and Quality Matter," RAND Journal of Economics, 41, 1-34.

Athey, S., D. Coey, And J. Levin (2013): "Set-Asides and Subsidies in Auctions," American Economic Journal: Microeconomics, 5, 1-27.

Border, K. C. (1991): "Implementation of Reduced Form Auctions: A Geometric Approach," Econometrica, 59, 1175-1187.

(2007): "Reduced Form Auctions Revisited," Economic Theory, 31, 167-181.

Brusco, S. And G. Lopomo (2002): "Collusion via Signalling in Simultaneous Ascending Bid Auctions with Heterogeneous Objects, with and without Complementarities," Review of Economics Studies, 69, 407-436.

Budish, E., Y.-K. Che, F. Kojima, and P. Milgrom (2013): "Designing Random Allocation Mechanisms: Theory and Applications," American Economic Review, 103, 585623.

Cai, Y., C. Daskalakis, and M. Weinberg (2011): "An Algorithmic Characterization of Multi-Dimensional Mechanisms," Working paper, MIT.

Che, Y.-K., D. Condorelli, And J. Kim (2012): "Weak Cartels and Optimal Auctions," Working paper.

${ }^{29}$ The argument leading to (22) goes through even if $T$ is not minimal among all violating sets $T \subset D$ because $T_{j} \backslash A$ and $T_{k} \backslash B$ are either upper contour sets or empty, respectively. 
Che, Y.-K., W. Dessein, And N. Kartik (2013): "Pandering to Persuade," American Economic Review, 103, 47-79.

Che, Y.-K. And I. Gale (1998): "Standard Auctions with Financially Constrained Bidders," Review of Economic Studies, 65, 1-21.

(2000): "The optimal Mechanism for Selling to a Budget-Constrained Buyer," Journal of Economic Theory, 92, 198-233.

Cramton, P., R. Gibbons, and P. Klemperer (1987): "Dissolving a Partnership Efficiently," Econometrica, 55, 615-632.

Edmonds, J. (1970): "Submodular Functions, Matroids, and Certain Polyhedra," Combinatorial Structures and Their Applications, R. Guy, H. Hanani, N. Sauer, and J. Schonheim, eds, Gordon and Breach, New York, 69-87.

Federgruen, A. And H. Groenevelt (1988): "Polymatroidal Flow Network Models with Multiple Sinks," Networks, 18, 285-302.

Frank, A. And E. Tardos (1988): "Generalized Polymatroids and Submodular Flows," Mathematical Programming, 42, 489-563.

Gale, D. (1957): "A Theorem on Flows in Networks," Pacific Journal of Mathematics, 7, 1073-1082.

Gershkov, A., J. K. Goeree, A. Kushnir, B. Moldovanu, and X. Shi (2013): "Bayesian and Dominant Strategy Implementation Revisited," Econometrica, 81, 197-220.

Goeree, J. K. And A. Kushnir (2011): "A Geometric Approach to Mechanism Design," Unpublished working paper, University of Zürich.

Gutmann, S., J. H. B. Kemperman, J. A. Reeds, and L. A. Shepp (1991): "Existence of Probability Measures with Given Marginals," Annals of Probability, 19, 1781-1797.

Hart, S. and P. Reny (2011): "Implementation of Reduced Form Auctions: A Simple Approach," Hebrew University of Jerusalem, unpublished working paper.

Hassin, R. (1982): "Minimum Cost Flow with Set-Constraints," Networks, 12, 1-21.

Hörner, J. And L. SAmuelson (2011): "Managing Strategic Buyers," Journal of Political Economy, 119, 379-425.

Kim, S. W., M. Olivares, and G. Y. Weintraub (2012): "Measuring the Performance of Large-Scale Combinatorial Auctions: A Structural Estimation Approach," Management Science, 58, 1458-1481.

LAfFont, J.-J. And J. Robert (1996): "Optimal Auctions with Financially Constrained Buyers," Economics Letters, 52, 181-186.

Manelli, A. And D. Vincent (2010): "Bayesian and Dominant-Strategy Implementation in the Independent Private Values Model," Econometrica, 78, 1905-1938.

Maskin, E. (2000): "Auctions, Development, and Privatization: Efficient Auctions with Liquidity-Constrained Buyers," European Economic Review, 44, 667-681. 
Maskin, E. And J. Riley (1984): "Optimal Auctions with Risk Averse Buyers," Econometrica, 52, 1473-1518.

Matthews, S. (1984): "On the Implementability of Reduced Form Auctions," Econometrica, 52, 1519-1522.

McAfee, P. And J. McMillan (1992): "Bidding Rings," American Economic Review, $82,579-599$.

MierendorfF, K. (2009): "Optimal Dynamic Mechanism Design with Deadlines," University of Bonn, unpulished working paper.

— (2011): "Asymmetric Reduced Form Auctions," Economics Letters, 110, 41-44.

Miralles, A. (2012): "Cardinal Baysian Allocation Mechanisms without Transfers," Journal of Economic Theory, 147, 179-206.

Myerson, R. B. (1981): "Optimal Auction Design," Mathematics of Operations Reseach, $6,58-63$.

PAI, M. (2012): "Competing Auctioneers," Unpublished manuscript, University of Pennsylvania.

PAi, M. AND R. Vohra (2011): "Optimal Auctions with Financially Constrained Bidders," Northwestern University, unpublished working paper.

- (2012): "Auction Design with Fairness Concerns: Subsidies vs. Set-Asides," Unpublished manuscript, University of Pennsylvania.

Parlane, S. (2001): "Contracting with Capacity Constrained Suppliers," Economic Theory, 17, 619-639.

Schrijver, A. (2000): Combinatorial Optimization: Polyhedra and Efficiency, Berlin Heidelberg New York: Springer-Verlag.

Shi, P. (2009): "Revenue Maximizing Auction when Bidders have Private Budgets," Unpublished manuscript, Duke University.

Vohra, R. (2011): Mechanism Design: A Linear Programming Approach, Cambridge University Press. 


\title{
Generalized Reduced-Form Auctions: A Network Flow Approach, Supplementary Material
}

\author{
By Yeon-Koo Che, Jinwoo Kim, and Konrad Mierendorff
}

\section{Appendix A. Omitted Proofs}

A.1. Structure of the Set of Reduced Form Auctions. We provide the proof of Theorem 4 in Remark 2 which shows that the two functions, $\Psi$ and $\Phi$, which set an upper bound and lower bound for the set of reduced form auctions, respectively, form a paramodular pair.

Proof of Theorem 4. We first observe that the operation $I(\theta, \cdot)$ as a function of $T$ preserves the union, intersection, and complement of sets: that is, for any $\theta \in \Theta$ and $T, T^{\prime} \subset D$, $I\left(\theta, T \cap T^{\prime}\right)=I(\theta, T) \cap I\left(\theta, T^{\prime}\right), I\left(\theta, T \cup T^{\prime}\right)=I(\theta, T) \cup I\left(\theta, T^{\prime}\right)$, and $I\left(\theta, T \backslash T^{\prime}\right)=I(\theta, T) \backslash$ $I\left(\theta, T^{\prime}\right)$. To see that the complement is preserved, for instance, note that $i \in I\left(\theta, T \backslash T^{\prime}\right)$ if and only if $\theta_{i} \in T \backslash T^{\prime}$, i.e., $\theta_{i} \in T$ and $\theta_{i} \notin T^{\prime}$, which is equivalent to having $i \in I(\theta, T)$ and $i \notin I\left(\theta, T^{\prime}\right)$, i.e., $i \in I(\theta, T) \backslash I\left(\theta, T^{\prime}\right)$. The other equalities can be checked similarly.

Given this, paramodularity of $\Psi$ and $\Phi$ holds due to the fact that the paramodularity of $C$ and $L$ is not affected by the expectation operator. For instance, the compliance holds since for any $T, T^{\prime} \subset D$,

$$
\begin{aligned}
\Psi\left(T^{\prime}\right)-\Phi(T) & =\sum_{\theta \in \Theta}\left[C\left(I\left(\theta, T^{\prime}\right)\right)-L(I(\theta, T))\right] p(\theta) \\
& \geq \sum_{\theta \in \Theta}\left[C\left(I\left(\theta, T^{\prime}\right) \backslash I(\theta, T)\right)-L\left(I(\theta, T) \backslash I\left(\theta, T^{\prime}\right)\right)\right] p(\theta) \\
& =\sum_{\theta \in \Theta}\left[C\left(I\left(\theta, T^{\prime} \backslash T\right)\right)-L\left(I\left(\theta, T \backslash T^{\prime}\right)\right)\right] p(\theta) \\
& =\Psi\left(T^{\prime} \backslash T\right)-\Phi\left(T \backslash T^{\prime}\right) .
\end{aligned}
$$

The first and last equalities follow from the fact that $\Psi(T)=\sum_{\theta \in Y(T)} C(I(\theta, T)) p(\theta)=$ $\sum_{\theta \in \Theta} C(I(\theta, T)) p(\theta)$ and $\Phi(T)=\sum_{\theta \in Y(T)} L(I(\theta, T)) p(\theta)=\sum_{\theta \in \Theta} L(I(\theta, T)) p(\theta)$ since, for any $\theta \in \Theta \backslash Y(T), I(\theta, T)=\emptyset$ so $C(I(\theta, T))=L(I(\theta, T))=0$. The next to last equality follows from the observation in the previous paragraph while the inequality from the compliance of $C$ and $L$. An analogous argument can be used to show the sub- and supermodularity of $\Psi$ and $\Phi$, respectively.

A.2. General Type Distributions. For the proof of Theorem 5 we denote the set of expost allocation rules that respect $(C, L)$ by $\mathcal{Q}_{0}(C, L)$ and the set of implementable interim allocation rules for given $(C, L)$ by $\mathcal{Q}(C, L)$. 
Proof of Theorem 5. Let $\Lambda: \mathcal{Q}_{0}(C, L) \rightarrow \mathcal{Q}(C, L)$ be the function that maps an ex-post allocation rule to its reduced form. Note that since $q \in \mathcal{Q}_{0}(C, L)$ is bounded and $\mu$ is a probability measure, $\mathcal{Q}_{0}(C, L)$ and $\mathcal{Q}(C, L)$ are subsets of the Hilbert space $L_{2}\left(\Theta, \mu, \mathbb{R}^{|I|}\right)$. Along the lines of Lemma 5.4 in Border (1991), one can show that $\mathcal{Q}_{0}(C, L)$ and $\mathcal{Q}(C, L)$ are weakly compact and the linear mapping $\Lambda$ is weakly continuous.

If $Q: \Theta \rightarrow[0, C(I)]^{|I|}$ satisfies $\left(B^{C}\right)$ it is bounded and hence there exists a sequence of simple functions $\left(Q^{n}: \Theta \rightarrow[0, C(I)]^{|I|}\right)_{n \in \mathbb{N}}$ with $Q_{i}^{n}(\theta)=Q_{i}^{n}\left(\theta_{i}\right)$, such that for $n \rightarrow \infty, Q^{n}$ converges uniformly to $Q$, and $Q^{1} \leq Q^{2} \leq Q^{3} \leq \ldots \leq Q$. Since convergence is uniform, there is a sequence $\left(\varepsilon_{n}\right)_{n \in \mathbb{N}}, \varepsilon_{n}>0$, such that $\varepsilon_{n} \rightarrow 0$ for $n \rightarrow \infty$, such for all $T=\left(T_{i}\right)_{i \in I}$, $T_{i} \in \mathcal{A}_{i}$,

$$
\int_{Y(T)} L_{\varepsilon_{n}}(I(\theta, T)) d \mu(\theta) \leq \sum_{i \in I} \int_{T_{i}} Q_{i}^{n}\left(\theta_{i}\right) d \mu_{i}\left(\theta_{i}\right) \leq \int_{Y(T)} C(I(\theta, T)) d \mu(\theta),
$$

where $L_{\varepsilon_{n}}(I(\theta, T))=\max \left\{L(I(\theta, T))-\varepsilon_{n}, 0\right\}$.

As $Q^{n}$ is a simple function we can write $Q_{i}^{n}$ as

$$
Q_{i}^{n}(\theta)=\sum_{k=1}^{K_{i}^{n}} \alpha_{i k}^{n} \chi_{A_{i k}^{n}}(\theta)
$$

where $\alpha_{i k}^{n} \in[0, C(I)],\left\{A_{i k}^{n}\right\}_{k}$ is a partition of $\Theta_{i}$ such that each $A_{i k}^{n} \in \mathcal{A}_{i}$, and $\chi_{A}$ is the indicator function of $A$.

Next, for given $n$ and each $i \in I$, we define a discretized type space $\tilde{\Theta}_{i}^{n}:=\left\{A_{i k}^{n}\right\}_{k=1, \ldots, K_{i}^{n}}$. The distribution over type profiles is given by

$$
\tilde{p}\left(A_{1 k_{1}}^{n}, \ldots, A_{|I| k_{|I|}}^{n}\right):=\mu\left(A_{1 k_{1}}^{n} \times \ldots \times A_{|I| k_{|I|}}^{n}\right) .
$$

Let $\tilde{Q}^{n}$ be the interim allocation rule for the discrete type-space $\Theta^{n}$ defined by

$$
\tilde{Q}_{i}^{n}\left(A_{i k}^{n}\right):=\alpha_{i k}^{n} .
$$

We have chosen $Q^{n}$ such that $\tilde{Q}^{n}$ is implementable for the relaxed constraints $\left(C, L-\varepsilon_{n}\right)$. Hence, for each $n$ there exists an allocation rule $\tilde{q}^{n}$, for the discrete type-space that respects $\left(C, L-\varepsilon_{n}\right)$ and has reduced form $\tilde{Q}^{n}$. Hence we can define an allocation rule $q^{n}$ for the continuous type space that respects $\left(C, L-\varepsilon_{n}\right)$ and has reduced form $Q^{n}$ : If $\theta \in A_{1 k_{1}}^{n} \times \ldots \times$ $A_{|I| k_{|I|}}^{n}$, we define

$$
q_{i}^{n}(\theta):=\tilde{q}_{i}^{n}\left(A_{1 k_{1}}^{n}, \ldots, A_{|I| k_{|I|}}^{n}\right) .
$$

So we have shown that $Q^{n} \in \mathcal{Q}\left(C, L-\varepsilon_{n}\right)$

Next, we take the limit $n \rightarrow \infty$ to show that $Q \in \mathcal{Q}(C, L)$. Since $q^{n} \in \mathcal{Q}_{0}(C, 0)$ for all $n$ and $\mathcal{Q}_{0}(C, L)$ is weakly compact, there is a weakly convergent subsequence with limit $q \in Q_{0}(C, L)$. Moreover, since $q^{n}$ respects $\left(C, L-\varepsilon_{n}\right)$ and $\varepsilon_{n} \rightarrow 0, q$ respects $(C, L)$, i.e., $q \in \mathcal{Q}_{0}(C, L)$. By continuity of $\Lambda$, there exists $Q^{\prime}$ such that $Q(\theta)=Q^{\prime}(\theta)$ for almost every $\theta$. 
Since $\mathcal{Q}(C, L)$ is a compact set, $Q^{\prime} \in \mathcal{Q}(C, L)$. As in the proof of Proposition 3.1 in Border (1991), one can show that also $Q \in \mathcal{Q}(C, L)$.

\section{A.3. Border Characterization in the Partitional Constraint Structure.}

Proof of Theorem 8. We first derive the effective constraints for arbitrary sets $G \subset I$. For any $G \subset I$, define

$$
\mathcal{H}_{G}^{L}:=\left\{G^{\prime} \in \tilde{\mathcal{H}} \mid G^{\prime} \subset G\right\} \text { and } \mathcal{H}_{G}^{C}:=\left\{G^{\prime} \in \tilde{\mathcal{H}} \mid G^{\prime} \cap G \neq \emptyset\right\} .
$$

First, we show that $C(G)=\phi\left(\mathcal{H}_{G}^{C}\right)=\min \left\{\sum_{G^{\prime} \in \mathcal{H}_{G}^{C}} C_{G^{\prime}}, C_{I}-\sum_{G^{\prime} \in \tilde{\mathcal{H}} \backslash \mathcal{H}_{G}^{C}} L_{G^{\prime}}\right\}$. To begin, observe that $C(G) \leq \phi\left(\mathcal{H}_{G}^{C}\right)$. This follows from the fact that for any $q \in \mathcal{P}$,

$$
\begin{aligned}
\sum_{i \in G} q_{i} & \leq \sum_{G^{\prime} \in \mathcal{H}_{G}^{C}} \sum_{i \in G^{\prime}} q_{i} \leq \sum_{G^{\prime} \in \mathcal{H}_{G}^{C}} C_{G^{\prime}} \\
\sum_{i \in G} q_{i} & \leq C_{I}-\sum_{i \in I \backslash G} q_{i} \leq C_{I}-\sum_{G^{\prime} \in \tilde{\mathcal{H}} \backslash \mathcal{H}_{G}^{C}} \sum_{i \in G^{\prime}} q_{i} \leq C_{I}-\sum_{G^{\prime} \in \tilde{\mathcal{H}} \backslash \mathcal{H}_{G}^{C}} L_{G^{\prime}},
\end{aligned}
$$

where the first inequality in (A.1) and the second inequality in (A.2) hold since $G \subset$ $\bigcup_{G^{\prime} \in \mathcal{H}_{G}^{C}} G^{\prime}$ and $q_{i} \geq 0, \forall i$. We construct an allocation $q \in \mathcal{P}$ to show that $\phi\left(\mathcal{H}_{G}^{C}\right)$ can be attained as a maximum of $(3)$, so $C(G)=\phi\left(\mathcal{H}_{G}^{C}\right)$. To this end, note that

$$
\begin{gathered}
\sum_{G^{\prime} \in \mathcal{H}_{G}^{C}} L_{G^{\prime}} \leq \phi\left(\mathcal{H}_{G}^{C}\right) \leq \sum_{G^{\prime} \in \mathcal{H}_{G}^{C}} C_{G^{\prime}}, \\
\phi\left(\mathcal{H}_{G}^{C}\right)+\sum_{G^{\prime} \in \tilde{\mathcal{H}} \backslash \mathcal{H}_{G}^{C}} L_{G^{\prime}} \leq C_{I} \leq \phi\left(\mathcal{H}_{G}^{C}\right)+\sum_{G^{\prime} \in \tilde{\mathcal{H}} \backslash \mathcal{H}_{G}^{C}} C_{G^{\prime}},
\end{gathered}
$$

which follows from the definition of $\phi$ and the assumption that $C_{G^{\prime}} \geq L_{G^{\prime}}, \forall G^{\prime} \in \tilde{\mathcal{H}}$ and $\sum_{G^{\prime} \in \tilde{\mathcal{H}}} L_{G^{\prime}} \leq L_{I} \leq C_{I} \leq \sum_{G^{\prime} \in \tilde{H}} C_{G^{\prime}}$. These two inequalities imply that there are $\lambda_{1}, \lambda_{2} \in$ $[0,1]$ such that

$$
\begin{gathered}
\phi\left(\mathcal{H}_{G}^{C}\right)=\sum_{G^{\prime} \in \mathcal{H}_{G}^{C}}\left[\lambda_{1} L_{G^{\prime}}+\left(1-\lambda_{1}\right) C_{G^{\prime}}\right], \\
C_{I}=\phi\left(\mathcal{H}_{G}^{C}\right)+\sum_{G^{\prime} \in \tilde{\mathcal{H}} \backslash \mathcal{H}_{G}^{C}}\left[\lambda_{2} L_{G^{\prime}}+\left(1-\lambda_{2}\right) C_{G^{\prime}}\right] .
\end{gathered}
$$

Now define $q$ as follows: for each $G^{\prime} \in \mathcal{H}_{G}^{C}, q_{i}=\frac{\lambda_{1} L_{G^{\prime}}+\left(1-\lambda_{1}\right) C_{G^{\prime}}}{\left|G \cap G^{\prime}\right|}$ if $i \in G^{\prime} \cap G$ while $q_{i}=0$ if $i \in G^{\prime} \backslash G$; for each $G^{\prime} \in \tilde{\mathcal{H}} \backslash \mathcal{H}_{G}^{C}$ and all $i \in G^{\prime}$, let $q_{i}=\frac{\lambda_{2} L_{G^{\prime}}+\left(1-\lambda_{2}\right) C_{G^{\prime}}}{\left|G^{\prime}\right|}$. Given this,

$$
\begin{aligned}
\sum_{i \in G} q_{i} & =\sum_{G^{\prime} \in \mathcal{H}_{G}^{C}} \sum_{i \in G \cap G^{\prime}} q_{i}=\sum_{G^{\prime} \in \mathcal{H}_{G}^{C}} \sum_{i \in G \cap G^{\prime}}\left(\frac{\lambda_{1} L_{G^{\prime}}+\left(1-\lambda_{1}\right) C_{G^{\prime}}}{\left|G \cap G^{\prime}\right|}\right)=\sum_{G^{\prime} \in \mathcal{H}_{G}^{C}}\left[\lambda_{1} L_{G^{\prime}}+\left(1-\lambda_{1}\right) C_{G^{\prime}}\right], \\
\sum_{i \in I \backslash G} q_{i} & =\sum_{G^{\prime} \in \mathcal{H}_{G}^{C}} \sum_{i \in G^{\prime} \backslash G} q_{i}+\sum_{G^{\prime} \in \tilde{\mathcal{H}} \backslash \mathcal{H}_{G}^{C}} \sum_{i \in G^{\prime}} q_{i}=\sum_{G^{\prime} \in \tilde{\mathcal{H}} \backslash \mathcal{H}_{G}^{C}} \sum_{i \in G^{\prime}} q_{i}=\sum_{G^{\prime} \in \tilde{\mathcal{H}} \backslash \mathcal{H}_{G}^{C}}\left[\lambda_{2} L_{G^{\prime}}+\left(1-\lambda_{2}\right) C_{G^{\prime}}\right] .
\end{aligned}
$$


Given (A.5) and (A.6), these equalities mean $\sum_{i \in G} q_{i}=\phi\left(\mathcal{H}_{G}^{C}\right)$ and $\sum_{i \in I} q_{i}=C_{I}$. Thus, it only remains to verify that $q \in \mathcal{P}$. The fact that $\sum_{i \in I} q_{i}=C_{I} \geq L_{I}$ means that the capacity constraints for $G=I$ is satisfied. For each $G^{\prime} \in \mathcal{H}_{G}^{C}$, we have $\sum_{i \in G^{\prime}} q_{i}=\lambda_{1} L_{G^{\prime}}+\left(1-\lambda_{1}\right) C_{G^{\prime}} \in$ $\left[L_{G^{\prime}}, C_{G^{\prime}}\right]$, so the capacity constraint is satisfied. Analogously, the capacity constraint is satisfied for each $G^{\prime} \in \tilde{\mathcal{H}} \backslash \mathcal{H}_{G}^{C}$.

Since establishing $L(G)=\psi\left(\mathcal{H}_{G}^{L}\right)$ is analogous, we only provide a sketch of proof. First, it is easy to see that $L(G) \geq \psi\left(\mathcal{H}_{G}^{L}\right)$, following a similar derivation as in (A.1) and (A.2). Also, (A.3) through (A.6) hold with $\phi, \mathcal{H}_{G}^{C}$, and $C_{I}$ being replaced by $\psi, \mathcal{H}_{G}^{L}$, and $L_{I}$, respectively, and with some $\lambda_{1}, \lambda_{2} \in[0,1]$. Construct an allocation $q \in \mathcal{P}$ which achieves $\psi\left(\mathcal{H}_{G}^{L}\right)$, as follows: for each $G^{\prime} \in \mathcal{H}_{G}^{L}$ and all $i \in G^{\prime}, q_{i}=\frac{\lambda_{1} L_{G^{\prime}}+\left(1-\lambda_{1}\right) C_{G^{\prime}}}{\left|G^{\prime}\right|}$; for each $G^{\prime} \in \tilde{\mathcal{H}} \backslash \mathcal{H}_{G}^{L}, q_{i}=$ $\frac{\lambda_{2} L_{G^{\prime}}+\left(1-\lambda_{2}\right) C_{G^{\prime}}}{\left|G^{\prime} \backslash G\right|}$ if $i \in G^{\prime} \backslash G$ while $q_{i}=0$ if $i \in G^{\prime} \cap G$. Given this, it is straightforward to see that $\sum_{i \in G} q_{i}=\sum_{G^{\prime} \in \mathcal{H}_{G}^{L}}\left[\lambda_{1} L_{G^{\prime}}+\left(1-\lambda_{1}\right) C_{G^{\prime}}\right]$ and $\sum_{i \in I \backslash G} q_{i}=\sum_{G^{\prime} \in \tilde{\mathcal{H}} \backslash \mathcal{H}_{G}^{L}}\left[\lambda_{2} L_{G^{\prime}}+\left(1-\lambda_{2}\right) C_{G^{\prime}}\right]$. The rest of the proof is parallel to that in the previous paragraph.

To summarize, we have shown that for any $G \subset I$, the effective constraints are given by $L(G)=\psi\left(\mathcal{H}_{G}^{L}\right)$ and $C(G)=\phi\left(\mathcal{H}_{G}^{C}\right)$. Lemma 1 implies that the effective constraints $(C, L)$ are paramodular. Now we are ready to prove the Theorem.

(i) Fix any $\theta=\left(\theta_{i}\right)_{i \in I}$, and define $T=\bigsqcup_{i \in I} T_{i}$ where $T_{i}=\left[\theta_{i}, \bar{\theta}_{i}\right]$. For any profile we have $C(I(\tilde{\theta}, T))=\phi\left(\mathcal{H}_{I(\tilde{\theta}, T)}^{C}\right)$. Inserting this into (the general type-space version) of $(B U)$ in Theorem 6 and noting that $C(I(\tilde{\theta}, T))=0$ if $\tilde{\theta} \notin Y(T)$, we get

$$
\begin{aligned}
\sum_{i \in I} \int_{\theta_{i}}^{\bar{\theta}_{i}} Q_{i}\left(s_{i}\right) d F_{i}\left(s_{i}\right) & \leq \int_{\Theta_{1}} \ldots \int_{\Theta_{|I|}} C(I(\tilde{\theta}, T)) d F_{1}\left(\tilde{\theta}_{1}\right) \ldots d F_{|I|}\left(\tilde{\theta}_{|I|}\right) \\
& =\sum_{\mathcal{H}^{\prime} \subset \mathcal{H}} \phi\left(\mathcal{H}^{\prime}\right) \operatorname{Pr}\left\{\mathcal{H}_{I(\tilde{\theta}, T)}^{C}=\mathcal{H}^{\prime}\right\} \\
& =\sum_{\mathcal{H}^{\prime} \subset \mathcal{H}} \phi\left(\mathcal{H}^{\prime}\right) \cdot \prod_{G \in \mathcal{H}^{\prime}}\left(1-\mathcal{F}_{G}(\theta)\right) \cdot \prod_{G \in \tilde{\mathcal{H}} \backslash \mathcal{H}^{\prime}} \mathcal{F}_{G}(\theta) .
\end{aligned}
$$

Meanwhile, consider $T=\bigsqcup_{i} T_{i}$ where $T_{i}=\left[\underline{\theta}_{i}, \theta_{i}\right]$. We have $L(I(\tilde{\theta}, T))=\psi\left(\mathcal{H}_{I(\tilde{\theta}, T)}^{L}\right)$. Inserting this into (the general type-space version) of $(B L)$ in Theorem 6 we have

$$
\begin{aligned}
\sum_{i \in I} \int_{\underline{\theta}_{i}}^{\theta_{i}} Q_{i}\left(s_{i}\right) d F_{i}\left(s_{i}\right) & \geq \int_{\Theta_{1}} \ldots \int_{\Theta_{|I|}} L(I(\tilde{\theta}, T)) d F_{1}\left(\theta_{1}\right) \ldots d F_{|I|}\left(\theta_{|I|}\right) \\
& =\sum_{\mathcal{H}^{\prime} \subset \mathcal{H}} \psi\left(\mathcal{H}^{\prime}\right) \operatorname{Pr}\left\{\mathcal{H}_{I(\tilde{\theta}, T)}^{L}=\mathcal{H}^{\prime}\right\} \\
& =\sum_{\mathcal{H}^{\prime} \subset \mathcal{H}} \psi\left(\mathcal{H}^{\prime}\right) \cdot \prod_{G \in \mathcal{H}^{\prime}} \mathcal{F}_{G}(\theta) \cdot \prod_{G \in \tilde{\mathcal{H}} \backslash \mathcal{H}^{\prime}}\left(1-\mathcal{F}_{G}(\theta)\right) .
\end{aligned}
$$

(ii) Last, the proof of (ii) follows from application of Corollary 3 to (i). 


\section{Appendix B. The Role of the Compliance Property}

The compliance condition ensures that the submodular upper bounds and supermodular lower bounds constitute effective bounds in the following sense:

Lemma 2 (Frank and Tardos, 1988, p. 502, Proposition 2.3). If $(C, L)$ is paramodular, then $C(G)=\max \left\{\sum_{i \in G} q_{i} \mid q=\left(q_{i}\right)_{i \in I}\right.$ respects $\left.(C, L)\right\}$ and $L(G)=\min \left\{\sum_{i \in G} q_{i} \mid q=\right.$ $\left(q_{i}\right)_{i \in I}$ respects $\left.(C, L)\right\}$ for each $G \subset I$.

Furthermore, there is a sense in which compliance constitutes a weakest sufficient condition or a maximal domain for submodular upper bounds and supermodular lower bounds to be effective. Note first that a violation of compliance can only occur for sets $G, G^{\prime} \subset I$ such that $G \cap G^{\prime} \neq \emptyset$, because otherwise $C\left(G^{\prime} \backslash G\right)-L\left(G \backslash G^{\prime}\right)=C\left(G^{\prime}\right)-L(G)$. Suppose that the four constraints $C\left(G^{\prime}\right), C\left(G^{\prime} \backslash G\right), L(G)$, and $L\left(G \backslash G^{\prime}\right)$ are given for sets $G, G^{\prime} \subset I$ with $G \cap G^{\prime} \neq \emptyset$, and compliance is violated for these sets. The following Lemma shows that if it is possible to extend the constraints to all subsets such that $C$ is submodular, $L$ is supermodular, and such that the set of feasible allocations is non-empty, then there exists such an extension for which at least one constraint is not effective.

Lemma 3. Let $G, G^{\prime} \in I$ with $G \cap G^{\prime} \neq \emptyset$ and let $C\left(G^{\prime}\right), C\left(G^{\prime} \backslash G\right), L(G), L\left(G \backslash G^{\prime}\right) \in \mathbb{R}_{+}$ such that $C\left(G^{\prime}\right)-L(G)<C\left(G^{\prime} \backslash G\right)-L\left(G \backslash G^{\prime}\right)$. If there exists an extension $(C(\tilde{G}), L(\tilde{G}))_{\tilde{G} \subset I}$ of these constraints to $2^{I}$, such that $C$ is submodular, $L$ is supermodular, and $\mathcal{P}:=\{x \in$ $\left.\mathbb{R}_{+}^{|I|} \mid L(\tilde{G}) \leq \sum_{i \in \tilde{G}} x_{i} \leq C(\tilde{G}), \forall \tilde{G} \subset I\right\} \neq \emptyset$, then there also exists an extension with these properties for which $C\left(G^{\prime} \backslash G\right)>\max \left\{\sum_{i \in G^{\prime} \backslash G} x_{i} \mid x \in \mathcal{P}\right\}$ or $L\left(G \backslash G^{\prime}\right)<\min \left\{\sum_{i \in G \backslash G^{\prime}} x_{i} \mid x \in\right.$ $\mathcal{P}\}$.

Proof. Note first that (a) if $C\left(G^{\prime}\right)<C\left(G^{\prime} \backslash G\right)$, then $C\left(G^{\prime} \backslash G\right)$ is not effective; (b) if $G \subset G^{\prime}$, the violation of compliance implies $C\left(G^{\prime} \backslash G\right)>C\left(G^{\prime}\right)-L(G)$ so that $C\left(G^{\prime} \backslash G\right)$ is not effective; and (c) if $G^{\prime} \subset G, L\left(G \backslash G^{\prime}\right)$ is ineffective because $L\left(G \backslash G^{\prime}\right)<L(G)-C\left(G^{\prime}\right)$. Hence the statement of the Lemma follows in all three cases.

Second, supermodularity of $L$ implies that $L$ is monotonic. Therefore, we can assume that $L(G) \geq L\left(G \backslash G^{\prime}\right)$ because otherwise no supermodular extension exists.

After these preliminary considerations, we only have to consider the case that $G \not \subset G^{\prime}$, $G^{\prime} \not \subset G, C\left(G^{\prime}\right) \geq C\left(G^{\prime} \backslash G\right)$, and $L(G) \geq L\left(G \backslash G^{\prime}\right)$. For this case we define $C\left(G \cap G^{\prime}\right)=$ $L\left(G \cap G^{\prime}\right)=C\left(G^{\prime}\right)-C\left(G^{\prime} \backslash G\right)$. Then the violation of compliance implies that $C\left(G \cap G^{\prime}\right)=$ $C\left(G^{\prime}\right)-C\left(G^{\prime} \backslash G\right)<L(G)-L\left(G \backslash G^{\prime}\right)$ and hence $L\left(G \backslash G^{\prime}\right)<L(G)-C\left(G \cap G^{\prime}\right)$, which means that $L\left(G \backslash G^{\prime}\right)$ is not effective.

The proof will be complete once we define $(C, L)$ for the remaining sets. We simplify notation by denoting $G_{1}=G^{\prime} \backslash G, G_{2}=G \backslash G^{\prime}$, and $G_{3}=G \cap G^{\prime}$. We fix a large number $K$ that is greater than the sum of all upper and lower bounds imposed on these sets and define 
for any $H \subset I$,

$$
C(H):= \begin{cases}\sum_{k \in\{1,3\}: G_{k} \cap H \neq \emptyset} C\left(G_{k}\right), & \text { if } H \subset G^{\prime} \\ K & \text { otherwise. }\end{cases}
$$

and

$$
L(H):= \begin{cases}L\left(G_{k}\right) & \text { if } \emptyset \neq G_{k} \subset H \text { for some } k \in\{2,3\} \text { and } G \nsubseteq H \\ L(G) & \text { if } G \subset H \\ 0 & \text { if } G_{k} \nsubseteq H \text { for all } k \in\{2,3\} .\end{cases}
$$

It is easy to check that the upper and lower bounds defined here are consistent with those given above. It is also easy to check that $C(H) \geq L(H)$ for any $H \subset I$ while both $C$ and $L$ are monotonic, i.e., $C(H) \leq C\left(H^{\prime}\right)$ for any $H \subset H^{\prime} \subset I$, and similarly for $L$. To see that $\mathcal{P}$ is nonempty, choose an element $i_{k} \in G_{k}$ for each $k=1,2,3$, and define $x \in \mathbb{R}_{+}^{|I|}$ by assigning $x_{i_{1}}=C\left(G^{\prime}\right)-C\left(G \cap G^{\prime}\right)=C\left(G^{\prime} \backslash G\right), x_{i_{2}}=K=C\left(G \backslash G^{\prime}\right) \geq L\left(G \backslash G^{\prime}\right)$, $x_{i_{3}}=L\left(G \cap G^{\prime}\right)=C\left(G \cap G^{\prime}\right)$, and $x_{i}=0$ for each $i \in I \backslash\left\{i_{1}, i_{2}, i_{3}\right\}$. It is then straightforward to verify that $x$ satisfies $(C, L)$ so $x \in \mathcal{P}$.

We next show that $C$ is submodular: for any two sets $H$ and $H^{\prime} \supset H$, and any $i \in$ $I \backslash H^{\prime}, C\left(H^{\prime} \cup\{i\}\right)-C\left(H^{\prime}\right) \leq C(H \cup\{i\})-C(H)$. This is immediate if $H^{\prime} \nsubseteq G^{\prime}$ or $i \notin G^{\prime}$ since in the former case, $C\left(H^{\prime} \cup\{i\}\right)=C\left(H^{\prime}\right)=K$ and $C(H \cup\{i\}) \geq C(H)$ while in the latter case, $C\left(H^{\prime} \cup\{i\}\right)=C(H \cup\{i\})=K$ and $C\left(H^{\prime}\right) \geq C(H)$. Thus we assume from now that $H \subset H^{\prime} \subset G^{\prime}$ and $i \in G^{\prime}$. Then, $i \in G_{k}$ for some $k=1,3$. If $H^{\prime} \cap G_{k}=\emptyset$, then $C\left(H^{\prime} \cup\{i\}\right)-C\left(H^{\prime}\right)=C\left(G_{k}\right)=C(H \cup\{i\})-C(H)$. If $H^{\prime} \cap G_{k} \neq \emptyset$, then $C\left(H^{\prime} \cup\{i\}\right)-C\left(H^{\prime}\right)=0 \leq C(H \cup\{i\})-C(H)$.

Lastly, we show that $L$ is supermodular: for any two sets $H$ and $H^{\prime} \supset H$, and any $i \in I \backslash H^{\prime}, L\left(H^{\prime} \cup\{i\}\right)-L\left(H^{\prime}\right) \geq L(H \cup\{i\})-L(H)$. Observe first that for any such $H \subset I$ and $i \in I$, we have $L(H \cup\{i\})-L(H)=0$ unless $G_{k} \nsubseteq H$ and $G_{k} \subset(H \cup\{i\})$ for some $k=2,3$, in which case we have either (i) $i \in G_{k}$ and $G_{k} \backslash\{i\} \subset H \cap G$ and $H \cap G \neq G \backslash\{i\}$ or (ii) $i \in G_{k}$ and $H \cap G=G \backslash\{i\}$. This implies that to show the supermodularity, it suffices to consider the two cases (i) and (ii). If (i) holds and $H^{\prime} \cap G \neq G \backslash\{i\}$, then $L(H \cup\{i\})-L(H)=L\left(G_{k}\right)=L\left(H^{\prime} \cup\{i\}\right)-L\left(H^{\prime}\right)$, as desired. If (i) holds and $H^{\prime} \cap G=G \backslash\{i\}$, then we have $G_{k} \nsubseteq H^{\prime}, G_{k^{\prime}} \subset H^{\prime}$ for $k^{\prime} \in\{2,3\} \backslash\{k\}$, and $G=G_{k} \cup G_{k^{\prime}} \subset H^{\prime} \cup\{i\}$, which implies $L(H \cup\{i\})-L(H)=L\left(G_{k}\right)<L(G)-L\left(G_{k^{\prime}}\right)=L\left(H^{\prime} \cup\{i\}\right)-L\left(H^{\prime}\right)$. Here the strict inequality follows from the fact that $L(G)>L\left(G \backslash G^{\prime}\right)+L\left(G \cap G^{\prime}\right)=L\left(G_{k}\right)+L\left(G_{k^{\prime}}\right)$. Finally, in case (ii) holds, we have $L(H \cup\{i\})-L(H)=L(G)-L\left(G_{k^{\prime}}\right)=L\left(H^{\prime} \cup\{i\}\right)-L\left(H^{\prime}\right)$, as desired.

\section{Appendix C. The Connection with Budish et Al. (2013)}

The characterization of feasible interim allocation rules we study has a connection with the characterization of the implementable expected allocations studied by Budish et al. 
(2013) (hereafter BCKM). BCKM study the constraint structure - the set of agent-object pairs whose assignment probability must obey some arbitrary integer-valued ceiling and floor constraints - that permits any expected assignment satisfying these constraints to be implemented by a lottery of deterministic assignments, each of which satisfies the same constraints. As mentioned in that paper, that requirement boils down to requiring that the set of feasible fractional assignments, which forms a bounded polytope, have integervalued extreme points. While both characterizations deal with implementability of some marginals via some joint distribution, there are several differences: (1) The integrality of the feasible set is the main issue in BCKM's characterization but it is not an issue in the current characterization, (2) our main challenge arises from the fact that there are different types of each agent, whereas no such problem arises in BCKM, and (3) BCKM adopt the notion of "universal implementation" which requires implementation to hold for all arbitrary quotas for the identified constraint structures. In contrast to this, we allow for arbitrary constraint structures but require the effective constraints to be paramodular. For the specific case of a hierarchical constraints structure, our Lemma 1 shows that paramodularity of the effective constraints is universal, i.e., it holds for arbitrary constraints on the hierarchical family. This is similar to BCKM, except their the corresponding condition is that the constraint sets form a pair of hierarchies.

Despite these differences, these two results have a common mathematical foundation, provided by the Edmonds' Polymatroid Intersection Theorem. This connection will also explain why the universal implementation in BCKM can be attained by bi-hierarchical constraint sets whereas it can be attained only by hierarchical constraint sets in the current context. For simplicity, we shall focus on the case in which the constraints are only in the upper bounds. This assumption can be dropped in most of the discussion, except for Section D.

To begin, let us define a polymatroid. Let $\Omega$ be a finite set, called the ground set, and consider a weight function $x: \Omega \rightarrow \mathbb{R}_{+}$. Let $\mathcal{X}$ denote all such functions. A bounded convex set

$$
\mathcal{P}=\left\{x \in \mathcal{X} \mid \sum_{\omega \in U} x(\omega) \leq f(U), \forall U \in 2^{\Omega}\right\}
$$

is said to be a polymatroid if $f: 2^{\Omega} \rightarrow \mathbb{R}_{+}$is submodular.

Edmonds' Polymatroid Intersection Theorem ${ }^{30}$ has the following two results:

Theorem 9. Let $\mathcal{P}$ and $\mathcal{P}^{\prime}$ be two polymatroids defined by $f$ and $f^{\prime}$.

(1) (Primal Integrality or PI): All extreme points of $\mathcal{P} \cap \mathcal{P}^{\prime}$ are integer-valued whenever $f$ and $f^{\prime}$ are integer-valued.

\footnotetext{
${ }^{30}$ See, for instance, Theorem 46.1 and Corollary 46.1a of Schrijver (2000)
} 
(2) (Total Dual Integrality or TDI): For any integer-valued n-vector $c$, the dual of maximizing $c^{T} x$ over $x \in \mathcal{P} \cap \mathcal{P}^{\prime}$, where $f$ and $f^{\prime}$ are rationals, has an integer optimal solution.

We now show how the characterizations given by these two papers relate to the two distinct parts of this theorem: BCKM relates to part 1 and our characterization relates to part 2 of Theorem 9 .

C.1. BCKM. It is easy to see how Theorem 9-1 implies the universal implementation characterization result of BCKM. In their model, the set $\Omega=N \times O$ is simply a set of agent-object pairs, with $N$ representing the set of agents and $O$ representing the set of objects, and for each $(i, o) \in \Omega$, the weight function $x(i, o)$ describes a (fractional) assignment of the object to agent $i$. BCKM then consider an arbitrary family $\mathcal{F} \subset 2^{\Omega}$ of subsets of $\Omega$, and require the fractional assignment to be in the set

$$
\mathcal{Q}:=\left\{x \in \mathcal{X} \mid \sum_{\omega \in U} x(\omega) \leq f(U), \forall U \in \mathcal{F}\right\} .
$$

Their universal implementation result then boils down to the statement that every extreme point of $\mathcal{Q}$ is integer-valued for any integer-valued $f$, if $\mathcal{F}$ comprises a pair of disjoint hierarchies, i.e., $\mathcal{F}=\mathcal{H} \cup \mathcal{H}^{\prime}$, where $\mathcal{H}$ and $\mathcal{H}^{\prime}$ are hierarchies. To see how Theorem 9-1 implies this statement, observe first that given the hypothesis

$$
\mathcal{Q}=\mathcal{P} \cap \mathcal{P}^{\prime}
$$

where $\mathcal{P}:=\left\{x \in \mathcal{X} \mid \sum_{\omega \in U} x(\omega) \leq f(U), \forall U \in \mathcal{H}\right\}$, and $\mathcal{P}^{\prime}:=\left\{x \in \mathcal{X} \mid \sum_{\omega \in U} x(\omega) \leq\right.$ $\left.f(U), \forall U \in \mathcal{H}^{\prime}\right\}$. To see now that the desired universal implementation characterization holds, it suffices to recall Lemma 1, which asserts that $\mathcal{P}$ and $\mathcal{P}^{\prime}$ (each set generated by quotas defined on hierarchical sets) are polymatroids. Hence, BCKM's main result follows from Theorem 9-1.

This perspective provides a new mathematical insight on BCKM. More interestingly, it suggests a way to extend BCKM. Suppose the assignment must satisfy upper bounds $f$ : $2^{\Omega} \rightarrow \mathbb{Z}_{+}$and lower bounds $g: 2^{\Omega} \rightarrow \mathbb{Z}_{+}$. We say that $(f, g)$ is bi-paramodular if there exist $\left(f_{1}, g_{1}\right)$ and $\left(f_{2}, g_{2}\right)$ such that $\left(f_{i}, g_{i}\right)_{i=1,2}$ is paramodular and $f=\min \left\{f_{1}, f_{2}\right\}$ and $g=\max \left\{g_{1}, g_{2}\right\}$. Then, we get the following result:

Theorem 10. Any fractional assignment $x$ is implementable with respect to $(f, g)$ if $(f, g)$ is bi-paramodular.

C.2. The current paper. The connection of Theorem 9 with the current paper is much more difficult to see, and so far, we have been able to establish it only for the upper bound case. The upshot is that at least in the case of upper bound only, we can see why Theorem 9-2 implies that the type of characterization like that in Theorem 3 should obtain. 
To begin, let $\tilde{q}_{i}(\theta)=q_{i}(\theta) p(\theta)$ and $\tilde{q}=\left(\tilde{q}_{i}(\theta)\right)_{i \in I, \theta \in \Theta}$. For any interim allocation rule $Q$, consider the following linear programming problem:

$$
\max _{\tilde{q} \geq 0} \sum_{i \in I, \theta \in \Theta} \tilde{q}_{i}(\theta)
$$

subject to

$$
\begin{array}{rlrl}
\sum_{i \in G} \tilde{q}_{i}(\theta) & \leq C(G) p(\theta), \forall G \subset I, \forall \theta \in \Theta, & & {[x(G, \theta)]} \\
\text { and } \sum_{\theta_{-i} \in \Theta_{-i}} \tilde{q}_{i}\left(\theta_{i}, \theta_{-i}\right) & \leq Q_{i}\left(\theta_{i}\right) p_{i}\left(\theta_{i}\right), \forall \theta_{i} \in \Theta_{i}, \forall i \in I, & {\left[z\left(i, \theta_{i}\right)\right]}
\end{array}
$$

where each variable in the square brackets is the dual variable for the corresponding constraint. The constraints (C.1) correspond to the capacity constraints we have in our model for subsets of agents. The constraints (C.2) correspond to the requirement that $Q$ is a reduced form (or implementable).

Note that given the last constraint, the optimal value of this problem cannot exceed the aggregate interim allocation probability, i.e., $\sum_{i \in I} \sum_{\theta_{i} \in \Theta_{i}} p_{i}\left(\theta_{i}\right) Q_{i}\left(\theta_{i}\right)$. Note also that the interim allocation rule $\left(Q_{i}\left(\theta_{i}\right)\right)_{\theta_{i} \in \Theta_{i}, i \in I}$ is a reduced form if and only if the optimal value equals $\sum_{i \in I} \sum_{\theta_{i} \in \Theta_{i}} p_{i}\left(\theta_{i}\right) Q_{i}\left(\theta_{i}\right)$.

To see how this program is related to our characterization, observe that the coefficients in the primal objective function are all 1's. Hence, if the feasible set associated with constraints (C.1) and (C.2) are TDI, then the dual of (P1) has an optimal integer solution, as implied by Theorem 9-2. It turns out that this implication gives rise to a Border type characterization, which will be established in the next section, Section D.

Hence, the important question, regarding our characterization, boils down to whether the feasible set associated with constraints (C.1) and (C.2) are TDI. The answer to this question is given by observing that each constraint gives rise to a polymatroid.

Lemma 4. Each of the constraints (C.1) and (C.2) gives rise to a polymatroid with $\Omega=I \times \Theta$ as a ground set.

Proof. Given the ground set $\Omega=I \times \Theta$, for each $\omega=(i, \theta) \in \Omega$ and $U \subset \Omega$, let $x(\omega)=\tilde{q}_{i}(\theta)$ and $x(U)=\sum_{\omega \in U} x(\omega)$.

We first show that the set of $\tilde{q}$ 's satisfying (C.1) is a polymatroid. To do so, define a weight function $f_{1}: 2^{\Omega} \rightarrow \mathbb{R}_{+}$as follow: For each $U \subset \Omega$, let $\alpha(\theta, U):=\{i \in I \mid(i, \theta) \in U\}$ and

$$
f_{1}(U)=\sum_{\theta \in \Theta} C(\alpha(\theta, U)) p(\theta) .
$$

Letting $\mathcal{P}_{1}:=\left\{x \in \mathbb{R}_{+}^{|\Omega|}: x(U) \leq f_{1}(U)\right\}$, it is straightforward to check that $\mathcal{P}_{1}$ is equivalent to the set of allocations satisfying (C.1), which is thus a polymatroid if $f_{1}$ is submodular. 
To show it, consider any subsets $U, U^{\prime} \subset \Omega$ with $U \subset U^{\prime}$ and any $\omega=(i, \theta) \notin U^{\prime}$. Then, we have $f_{1}(U \cup\{\omega\})-f_{1}(U)=[C(\alpha(\theta, U) \cup\{i\})-C(\alpha(\theta, U))] p(\theta) \geq\left[C\left(\alpha\left(\theta, U^{\prime}\right) \cup\{i\}\right)-\right.$ $\left.C\left(\alpha\left(\theta, U^{\prime}\right)\right)\right] p(\theta)=f_{1}\left(U^{\prime} \cup\{\omega\}\right)-f_{1}\left(U^{\prime}\right)$, where the inequality holds due to the fact that $\alpha(\theta, U) \subset \alpha\left(\theta, U^{\prime}\right)$ and $C$ is submodular.

We next show that the set of $\tilde{q}$ 's satisfying (C.2) is a polymatroid. To do so, define another weight function $f_{2}: 2^{\Omega} \rightarrow \mathbb{R}_{+}$as follow: For each $U \subset \Omega$, let $\left(i, \theta_{i}, \Theta_{-i}\right)=\left\{\left(i, \theta_{i}, \theta_{-i}\right): \theta_{-i} \in\right.$ $\left.\Theta_{-i}\right\}$ (by some abuse of notation) and

$$
f_{2}(U)=\sum_{\left(i, \theta_{i}\right):\left(i, \theta_{i}, \Theta_{-i}\right) \cap U \neq \emptyset} p_{i}\left(\theta_{i}\right) Q_{i}\left(\theta_{i}\right) .
$$

Letting $\mathcal{P}_{2}:=\left\{x \in \mathbb{R}_{+}^{|\Omega|}: x(U) \leq f_{2}(U)\right\}$, it is again straightforward to check that $\mathcal{P}_{2}$ is equivalent to the set of allocations satisfying (C.2), which is thus a polymatroid if $f_{2}$ is submodular. To show it, consider any subsets $U, U^{\prime} \subset \Omega$ with $U \subset U^{\prime}$ and any $\omega=$ $\left(i, \theta_{i}, \theta_{-i}\right) \notin U^{\prime}$. If $\left(i, \theta_{i}, \Theta_{-i}\right) \cap U \neq \emptyset$, then we have $f_{2}(U \cup\{\omega\})-f_{2}(U)=0=f_{2}\left(U^{\prime} \cup\right.$ $\{\omega\})-f_{2}\left(U^{\prime}\right)$. If $\left(i, \theta_{i}, \Theta_{-i}\right) \cap U=\emptyset$ and $\left(i, \theta_{i}, \Theta_{-i}\right) \cap U^{\prime} \neq \emptyset$, then $f_{2}\left(U^{\prime} \cup\{\omega\}\right)-f_{2}\left(U^{\prime}\right)=$ $0 \leq p_{i}\left(\theta_{i}\right) Q_{i}\left(\theta_{i}\right)=f_{2}(U \cup\{\omega\})-f_{2}(U)$. If $\left(i, \theta_{i}, \Theta_{-i}\right) \cap U^{\prime}=\emptyset$, then $f_{2}(U \cup\{\omega\})-f_{2}(U)=$ $p_{i}\left(\theta_{i}\right) Q_{i}\left(\theta_{i}\right)=f_{2}\left(U^{\prime} \cup\{\omega\}\right)-f_{2}\left(U^{\prime}\right)$.

Remark 4. (Universal Implementation). When the sets of agents facing quota constraints form a hierarchy, we have an universal implementation in the sense that regardless of the specific values of the quotas, the Border type characterization, specifically Theorem 3 , holds. The reason for this is that by Lemma 1, the quota constraints (C.1) form a polymatroid regardless of the specific values of the quotas. The reason that we cannot accommodate more (e.g., bihierarchy), as also proven by Remark 1, is because we have already used up another polymatroid in our reduced-form requirement (C.2). This is precisely the reason why bihierarchy is possible under BCKM but not in our case; they do not face additional constraints such as (C.2) that we have to deal with.

\section{Appendix D. Polymatroid Method for the Border Characterization}

In this subsection, we show that the polymatroid optimization problem stated in $(\mathrm{P} 1)$ provides an alternative way to obtain the Border characterization. As mentioned earlier, this result is established by using the fact that the constraints of (P1) are TDI so the dual problem has an integer solution. For this argument, we need to assume that $p$ and $Q$ are all rational numbers. We note that the argument below is not readily adaptable to the general case with both upper and lower bound constraints. This illustrates the advantage of using our network flow approach to obtain the generalized characterization as in Theorem 3. 
To begin, let us write the dual problem to (P1) as follows:

$$
\min _{x(\cdot), z(\cdot)} \sum_{G \subset I, \theta \in \Theta} p(\theta) C(G) x(G, \theta)+\sum_{i \in I} \sum_{\theta_{i} \in \Theta_{i}}\left[Q_{i}\left(\theta_{i}\right) p_{i}\left(\theta_{i}\right) z\left(i, \theta_{i}\right)\right]
$$

subject to

$$
\sum_{G: i \in G} x(G, \theta)+z\left(i, \theta_{i}\right) \geq 1, \forall i \in I, \forall \theta \in \Theta
$$

and $x(G, \theta), z\left(i, \theta_{i}\right) \geq 0, \forall G, \theta, i, \theta_{i}$. To show the sufficiency of the Border condition for implementability of $Q,{ }^{31}$ suppose that $Q$ is not a reduced form, which means that the optimal value of the primal, and thus the dual, problem is smaller than $\sum_{i \in I} \sum_{\theta_{i} \in \Theta_{i}} p_{i}\left(\theta_{i}\right) Q_{i}\left(\theta_{i}\right)$. We show that this leads to the violation of upper bound condition in $\left(B^{\prime}\right)$ for some $T \subset D .{ }^{32}$

To this end, recall first that the constraints of (P1) are TDI, so its dual (Dual-1) has an integer solution, which then implies $z\left(i, \theta_{i}\right)=0$ or 1 for all $\left(i, \theta_{i}\right)$, since otherwise one could reduce $z\left(i, \theta_{i}\right)$, and thereby the value of the objective function, without violating (D.1).

Given any such optimal $z(\cdot)$, the dual problem (Dual-1) can be decomposed into the following sub-problems: for each $\theta \in \Theta$,

$$
\min _{x(\cdot, \theta), y(\cdot, \theta)} p(\theta) \sum_{G \subset I} C(G) x(G, \theta)
$$

subject to

$$
\sum_{G: i \in G} p(\theta) x(G, \theta) \geq p(\theta)\left[1-z\left(i, \theta_{i}\right)\right], \forall i \in I .
$$

With $\gamma(i, \theta)$ denoting the dual variable for the constraint (D.2), the dual problem to (Dual-2) can be written as

$$
\max _{\gamma(\cdot, \theta)} \sum_{i \in I} p(\theta)\left[1-z\left(i, \theta_{i}\right)\right] \gamma(i, \theta)
$$

subject to

$$
\sum_{i \in G} \gamma(i, \theta) \leq C(G), \forall G \subset I
$$

To solve $(\mathrm{P} 2)$, let $T_{i}=\left\{\theta_{i} \in \Theta_{i} \mid z\left(i, \theta_{i}\right)=0\right\}$ for each $i \in I$, so $z\left(i, \theta_{i}\right)=1$ for any $\theta_{i} \in \Theta_{i} \backslash T_{i}$. Recall that with $T=\bigsqcup_{i \in I} T_{i}, I(\theta, T)=\left\{i \in I \mid \theta_{i} \in T_{i}\right\}$. Then, the objective function of (P2) becomes

$$
\sum_{i: z\left(i, \theta_{i}\right)=0} p(\theta) \gamma(i, \theta)=p(\theta) \sum_{i \in I(\theta, T)} \gamma(i, \theta),
$$

\footnotetext{
${ }^{31}$ The proof of necessity is straightforward and thus omitted.

${ }^{32}$ The duality argument we use below is similar to that in Cai et al. (2011). Unlike Cai et al. (2011), however, our argument exploits the TDI property to yield the Border characterization, which is much tighter than the characterization in Cai et al. (2011).
} 
which clearly attains its maximum when $\sum_{i \in I(\theta, T)} \gamma(i, \theta)=C(I(\theta, T))$, given the constraint (D.3). Plug this into the objective function of (Dual-1) to obtain

$$
\sum_{\theta \in \Theta} p(\theta) C(I(\theta, T))+\sum_{i \in I} \sum_{\theta_{i} \in \Theta_{i}} p_{i}\left(\theta_{i}\right) Q_{i}\left(\theta_{i}\right) z\left(i, \theta_{i}\right)
$$

Now that this expression must be smaller than $\sum_{i \in I} \sum_{\theta_{i} \in \Theta_{i}} p_{i}\left(\theta_{i}\right) Q_{i}\left(\theta_{i}\right)$ by assumption, we get

$$
\begin{aligned}
0 & >\sum_{\theta \in \Theta} p(\theta) C(I(\theta, T))+\sum_{i \in I} \sum_{\theta_{i} \in \Theta_{i}} p_{i}\left(\theta_{i}\right) Q_{i}\left(\theta_{i}\right)\left[z\left(i, \theta_{i}\right)-1\right] \\
& =\sum_{\theta \in Y(T)} p(\theta) C(I(\theta, T))-\sum_{i \in I} \sum_{\theta_{i} \in T_{i}} p_{i}\left(\theta_{i}\right) Q_{i}\left(\theta_{i}\right)
\end{aligned}
$$

which means that $\left(B^{\prime}\right)$ is violated for $T$, as desired.

D.1. A characterization for general constraints. Without assuming supermodularity of the upper bounds, Cai et al. (2011) derive a characterization the involves a continuum of constraints. To state their result we define

$$
\mathcal{A}(C):=\left\{x \in[0,1]^{|I|} \mid \sum_{i \in G} x_{i} \leq C(G), \forall G \subset I\right\}
$$

as the set of allocations that is feasible for given upper bounds $C: 2^{I} \rightarrow[0, n]$. In the following theorem, $C$ need not be submodular.

Theorem 11 (Cai et al., 2011). Let $Q$ be an interim allocation rule. $Q$ is the reduced form of an allocation rule that respects $(C, 0)$ if and only if for all weights $\left(W_{i}\left(\theta_{i}\right)\right)_{i \in I, \theta_{i} \in \Theta_{i}} \in$ $[0,1]^{\sum_{i}\left|\Theta_{i}\right|}$

$$
\sum_{i \in I} \sum_{\theta_{i} \in \Theta_{i}} W_{i}\left(\theta_{i}\right)\left[p_{i}\left(\theta_{i}\right) Q_{i}\left(\theta_{i}\right)\right] \leq \sum_{\theta \in \Theta} \max _{x \in \mathcal{A}(\mathcal{C})}\left\{\sum_{i \in I} W_{i}\left(\theta_{i}\right) x_{i}\right\}
$$

This characterization is obtained from the dual linear program (Dual-1) and the weights $W$ are the dual variables $z$. Therefore, submodularity implies that (D.4) has to be checked only for integer-valued weights. But for $\left(W_{i}\left(\theta_{i}\right)\right)_{i \in I, \theta_{i} \in \Theta_{i}} \in\{0,1\}^{\sum_{i}\left|\Theta_{i}\right|}$ (D.4) is equivalent to $\left(B^{\prime}\right)$ with $T=\left\{\theta_{i} \in D \mid W_{i}\left(\theta_{i}\right)=1\right\}$.

Conversely, if submodularity is violated, some of the constraints in (D.4) induced by noninteger weights are binding. To see this, consider the first example in Table I in Remark 1. If we maximize the objective function subject to $\left(B^{\prime}\right)$, a maximizer is given by $Q_{i}^{*}\left(\underline{\theta}_{i}\right)=13 / 8$ and $Q_{i}^{*}\left(\bar{\theta}_{i}\right)=9 / 4$ for all $i \in I$. For this interim allocation rule, (D.4) is for example violated for weights $W_{i}\left(\underline{\theta}_{i}\right)=1 / 2$ and $W_{i}\left(\bar{\theta}_{i}\right)=1$ for all $i \in I$. Indeed, a straightforward calculation shows that for these weights and the interim allocation rule $Q^{*}$, the LHS of (D.4) is 147/32 whereas the RHS is $9 / 2$, which is strictly smaller. This demonstrates that the additional 
constraints can in general not be neglected and the characterization obtained in the absence of submodularity is much less tractable than our characterization in Theorem 3.

\section{REFERENCES}

Border, K. C. (1991): "Implementation of Reduced Form Auctions: A Geometric Approach," Econometrica, 59, 1175-1187.

Budish, E., Y.-K. Che, F. Kojima, And P. Milgrom (2013): "Designing Random Allocation Mechanisms: Theory and Applications," American Economic Review, 103, 585623.

Cai, Y., C. Daskalakis, And M. Weinberg (2011): "An Algorithmic Characterization of Multi-Dimensional Mechanisms," Working paper, MIT.

Frank, A. And E. TARdos (1988): "Generalized Polymatroids and Submodular Flows," Mathematical Programming, 42, 489-563. 